\title{
Spectral element modeling of spontaneous earthquake rupture on rate and state faults: Effect of velocity-strengthening friction at shallow depths
}

\author{
Y. Kaneko, ${ }^{1}$ N. Lapusta, ${ }^{1,2}$ and J.-P. Ampuero ${ }^{1}$ \\ Received 17 December 2007; revised 2 June 2008; accepted 11 June 2008; published 27 September 2008.
}

[1] We develop a spectral element method (SEM) for simulating dynamic rupture on rate and state faults and use it to study how the rupture is affected by a shallow fault region of steady state velocity-strengthening friction. Our comparison of the developed SEM and a spectral boundary integral method (BIM) for an antiplane (two-dimensional) test problem shows that for the finest resolution that we use, the two methods produce virtually identical solutions, with negligible differences in rupture arrival times and peak slip velocities (less than $0.05 \%$ of their absolute values). The convergence with grid reduction of the developed SEM is comparable to that of BIM. We also use the test problem to compare numerical resolution required for different state evolution laws and for linear slip-weakening friction. Using our three-dimensional implementation of the methodology, we find that a shallow velocity-strengthening fault region can significantly alter dynamic rupture and ground motion. The velocity-strengthening region suppresses supershear propagation at the free surface occurring in the absence of such region, which could explain the lack of universally observed supershear rupture near the free surface. In addition, the velocity-strengthening region promotes faster falloff of slip velocity behind the rupture front and decreases final slip throughout the entire fault, causing a smaller average stress drop. The slip decrease is largest in the shallow parts of the fault, resulting in a depth profile of slip qualitatively consistent with observations of shallow coseismic slip deficit. The shallow velocity-strengthening region also reduces the amplification of strong ground motion due to a low-velocity bulk structure.

Citation: Kaneko, Y., N. Lapusta, and J.-P. Ampuero (2008), Spectral element modeling of spontaneous earthquake rupture on rate and state faults: Effect of velocity-strengthening friction at shallow depths, J. Geophys. Res., 113, B09317, doi:10.1029/2007JB005553.

\section{Introduction}

[2] Understanding complex and realistic scenarios of seismic and aseismic slip demands accurate and efficient numerical models that incorporate appropriate fault constitutive laws. Rate- and state-dependent friction laws have been widely used to simulate and explain various earthquake phenomena including earthquake nucleation, postseismic slip, foreshocks, aftershocks, and aseismic transients [e.g., Marone et al., 1991; Dieterich, 1994; Ben-Zion and Rice, 1997; Marone, 1998; Lapusta and Rice, 2003; Liu and Rice, 2005; Dieterich, 2007, and references therein; Kaneko and Lapusta, 2008]. A common approach to model slip on a rate and state (RS) fault is to employ boundary integral methods (BIMs). In BIMs, field quantities are considered

\footnotetext{
${ }^{1}$ Division of Geological and Planetary Sciences, California Institute of Technology, Pasadena, USA.

${ }^{2}$ Division of Engineering and Applied Science, California Institute of Technology, Pasadena, USA.

Copyright 2008 by the American Geophysical Union. 0148-0227/08/2007JB005553\$09.00
}

only at the boundary of a domain, and integral expressions are used to account for elastic interactions with the surrounding media. In the framework of BIM, nucleation, rupture propagation, and arrest of earthquakes have been successfully modeled [e.g., Ben-Zion and Rice, 1997; Lapusta et al., 2000]. However, these studies have been mostly restricted to planar faults embedded into a uniform elastic space. At the same time, observations point to complicated crustal structures with variable bulk properties, fault damage zones, and nonplanar fault geometries. It is important to include those factors into earthquake models, combining them with laboratory-derived constitutive fault relations such as RS friction.

[3] Finite element methods (FEMs) and, in particular, spectral element methods (SEMs), can incorporate variable bulk properties and more complex fault geometries. They have been used to simulate single-earthquake scenarios, mostly with linear slip weakening (LSW) friction [e.g., Oglesby et al., 1998; Aagaard et al., 2001; Ampuero, 2002; Festa and Vilotte, 2006; Madariaga et al., 2006]. The application of SEM to wave propagation problems is well developed [Komatitsch and Vilotte, 1998; Komatitsch and Tromp, 1999] and has been recently reviewed by 
Komatitsch et al. [2005] and Chaljub et al. [2007]. SEMs combine the flexibility of FEMs with high numerical accuracy due to the use of higher-order Lagrange interpolants on Gauss-Lobatto-Legendre (GLL) points that mimic the behavior of the Legendre basis [Komatitsch and Vilotte, 1998; Komatitsch and Tromp, 1999]. Furthermore, the SEMs with a diagonal mass matrix reduce computational costs associated with solving a large linear system and result in relatively simple parallel implementation. The method is well suited for describing surfaces of displacement discontinuity with mixed traction-displacement interface conditions (e.g., faults) by the split node technique, as in FEMs [Oglesby et al., 1998; Aagaard et al., 2001] and some finite difference methods [Andrews, 1999; Day et al., 2005]. Fault surfaces with the split node technique have been implemented in 2-D SEMs [Ampuero, 2002; Vilotte et al., 2006], and SEMs with the split node technique have been successfully applied to wave propagation across compliant faults [Haney et al., 2007] and to earthquake source dynamics [Festa and Vilotte, 2005; Madariaga et al., 2006].

[4] In section 2, we present a SEM algorithm for modeling dynamic rupture on a RS fault. The 3-D SEM we use was originally developed for wave propagation by Komatitsch and Tromp [1999]; our work is an extension of the study by Ampuero [2002] that incorporated a LSW fault boundary into that SEM framework. We have extended the formulation to RS faults. To validate the developed SEM approach, we have conducted detailed comparison of SEM and BIM simulation results obtained for an antiplane problem (section 3). Incorporating RS faults into a SEM formulation requires a semi-implicit numerical scheme which makes the implementation more challenging than that for LSW friction.

[5] Using the developed formulation, we have investigated the effect of velocity-strengthening fault friction in the shallow portion of a fault on dynamic rupture scenarios (section 4). Accumulating evidence supports the presence of velocity-strengthening friction at shallow depths. In laboratory experiments, rock friction at low normal stress typically exhibits velocity-strengthening behavior due to unconsolidated fault gouge [e.g., Marone et al., 1991; Marone, 1998]. Theoretical studies have shown that velocity-strengthening friction responds to loading with stable sliding and does not allow for spontaneous nucleation of frictional instabilities [Ruina, 1983; Rice and Ruina, 1983]. Hence, studies of interseismic shallow creep [e.g., Lyons et al. 2002], shallow afterslip of large earthquakes [e.g., Marone et al., 1991; Marone, 1998; Hsu et al., 2006], and the deficit of seismicity at shallow depths [e.g., Shearer et al. 2005] provide indirect observational evidence for velocity-strengthening fault rheology at shallow depths. It is important to understand how velocity-strengthening friction at shallow depths affects earthquake rupture dynamics and, as a consequence, ground motion and seismic hazard assessment in the vicinity of active faults. Furthermore, the shallow velocitystrengthening region may also be relevant for tsunami earthquakes in subduction zones as it may slow down the updip propagation of rupture, boost the low-frequency content, and promote tsunami generation [Polet and Kanamori, 2000; Seno, 2002].

[6] In addition, a typical Earth bulk structure has strong variation of elastic parameters with depth. The reduction of elastic moduli near the free surface results in ground motion amplification, and thus it has important consequences for seismic hazard [e.g., Olsen 2000]. Such bulk variations cannot be accommodated with existing BIM formulations, while SEM can incorporate them with ease. We have simulated dynamic rupture scenarios on a fault embedded in a layered bulk structure and studied how the peak ground motion at on- and off-fault sites is affected by the bulk structure combined with different fault rheologies (section 5).

\section{A SEM Algorithm for Simulations of Dynamic Rupture on Rate and State Faults}

\subsection{Discretized Elastodynamic Relations}

[7] In SEM, the geometry of the mesh elements is represented by the product of low-degree Lagrange polynomials like in the classical FEM, while the field approximation function is represented by the product of highdegree Lagrange polynomials defined on the GaussLobatto-Legendre (GLL) points. The choice of coincident nodes for interpolation and numerical integration results in a diagonal mass matrix of the discretized domain, reducing the computational costs associated with solving a large linear system and making its parallel implementation relatively simple.

[8] The discretization of the weak form of the equation of motion leads to the matrix equation:

$$
\mathbf{M} \ddot{\mathbf{u}}=-\mathbf{K} \mathbf{u}+\mathbf{B} \tau,
$$

where $\mathbf{M}$ and $\mathbf{K}$ are the mass and stiffness matrix respectively, given by Komatitsch et al. [2005] (equation (57) and (59)), the fault boundary matrix $\mathbf{B}$ is described in Appendix A, $\tau=\mathbf{T}-\tau_{o}$ is the relative traction vector on the fault, $\mathbf{T}$ is the total traction, and $\tau_{o}$ is the traction on the fault that corresponds to the reference zero displacement state. Vectors $\mathbf{u}, \dot{\mathbf{u}}$, and $\ddot{\mathbf{u}}$ collect the values of displacements, particle velocities, and accelerations, respectively, of all the computational nodes of the bulk mesh.

[9] Our time discretization scheme is based upon the explicit acceleration Newmark scheme:

$$
\begin{gathered}
\mathbf{u}_{n+1}=\mathbf{u}_{n}+\Delta t \dot{\mathbf{u}}_{n}+\frac{\Delta t^{2}}{2} \ddot{\mathbf{u}}_{n}, \\
\mathbf{M} \ddot{\mathbf{u}}_{n+1}=-\mathbf{K} \mathbf{u}_{n+1}+\mathbf{B} \tau_{n+1}, \\
\dot{\mathbf{u}}_{n+1}=\dot{\mathbf{u}}_{n}+\Delta t \frac{\ddot{\mathbf{u}}_{n}+\ddot{\mathbf{u}}_{n+1}}{2},
\end{gathered}
$$

where the subscripts $n$ and $n+1$ refer to the number of the time step. Given all the quantities at the $n$th time step, we would like to obtain the quantities at the $(n+1)$ th time step. The nontrivial advance is to obtain $\tau_{n+1}$ in (3) on the fault simultaneously with fault constitutive relations. Combining (3) and (4), one obtains

$$
\dot{\mathbf{u}}_{n+1}=\dot{\mathbf{u}}_{n+1}^{\text {free }}+\frac{\Delta t}{2} \mathbf{M}^{-1} \mathbf{B} \tau_{n+1},
$$




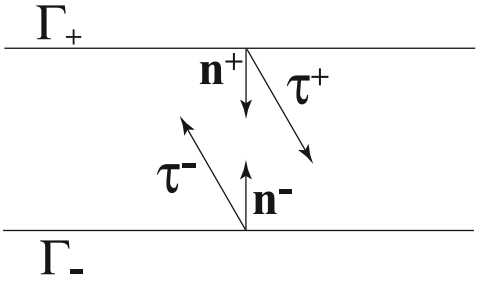

Figure 1. The fault divided into two nonoverlapping surfaces $\Gamma_{ \pm}$.

where

$$
\dot{\mathbf{u}}_{n+1}^{\text {free }} \equiv \dot{\mathbf{u}}_{n}+\frac{\Delta t}{2}\left(\ddot{\mathbf{u}}_{n}-\mathbf{M}^{-1} \mathbf{K} \mathbf{u}_{n+1}\right)
$$

is the "free velocity" that would prevail if the traction on the fault suddenly vanished, creating free boundary conditions. We now write relation (5) for the fault nodes with the \pm signs indicating the values of field variables on the two sides of the fault (Figure 1):

$$
\dot{\mathbf{u}}_{n+1}^{ \pm}=\dot{\mathbf{u}}_{n+1}^{\text {free } \pm}+\frac{\Delta t}{2} \mathbf{M}_{ \pm}^{-1} \mathbf{B}_{ \pm} \tau_{n+1}^{ \pm}
$$

Subtracting the minus side from the plus side, and using the sign convention $\tau=-\tau^{+}=\tau^{-}$, where $\tau^{ \pm}$are defined with respect to the outward normal from the fault boundary $\Gamma_{ \pm}$ (Figure 1), we obtain

$$
\dot{\boldsymbol{\delta}}_{n+1}=\dot{\boldsymbol{\delta}}_{n+1}^{\text {free }}-\mathbf{Z}^{-1} \boldsymbol{\tau}_{n+1}
$$

where vectors $\delta, \dot{\delta}$, and $\ddot{\delta}$ refer to the slip, slip velocity, and slip acceleration, defined as the difference between values of displacement, velocity, and acceleration, respectively, of corresponding split nodes across the fault plane (e.g., $\boldsymbol{\delta}=\mathbf{u}^{+}-\mathbf{u}^{-}$and $\dot{\boldsymbol{\delta}}^{\text {free }}=\dot{\mathbf{u}}^{\text {free+}}-\dot{\mathbf{u}}^{\text {free- }}$ ), and $\mathbf{Z}$ is the fault impedance matrix given by

$$
\mathbf{Z}^{-1} \equiv \frac{\Delta t}{2}\left(\mathbf{M}_{+}^{-1} \mathbf{B}_{+}+\mathbf{M}_{-}^{-1} \mathbf{B}_{-}\right)
$$

Note that for the cases that we consider in this study, the faultnormal component of traction $\mathbf{T}$ remains unchanged, and hence the fault-normal components of $\boldsymbol{\tau}$ and $\dot{\delta}$ are zero. The matrices $\mathbf{M}$ and $\mathbf{Z}^{-1}$ are diagonal, and $\mathbf{B}_{+}=\mathbf{B}_{\text {_ }}$ for conformal meshes. Solving (8) for $\tau_{n+1}$ gives

$$
\tau_{n+1}=\mathbf{Z} \dot{\delta}_{n+1}^{\text {free }}-\mathbf{Z} \dot{\boldsymbol{\delta}}_{n+1}
$$

Expression (10) is a local relation which can be solved node by node on the fault. It is convenient to rewrite (10) in terms of total traction, $\mathbf{T}=\boldsymbol{\tau}+\boldsymbol{\tau}_{o}$ :

$$
\begin{aligned}
\mathbf{T}_{n+1} & =\boldsymbol{\tau}_{o}+\mathbf{Z} \dot{\boldsymbol{\delta}}_{n+1}^{\text {free }}-\mathbf{Z} \dot{\boldsymbol{\delta}}_{n+1} \\
& \equiv \tilde{\mathbf{T}}_{n+1}-\mathbf{Z} \dot{\boldsymbol{\delta}}_{n+1},
\end{aligned}
$$

where $\tilde{\mathbf{T}}$ is the "stick traction" that would prevail if there were a sudden slip arrest.

\subsection{Rate and State Friction Laws}

[10] Rate- and state-dependent friction laws were developed to incorporate observations of rock friction experiments at relatively low sliding rates of $10^{-8}$ to $10^{-3} \mathrm{~m} / \mathrm{s}$ [Dieterich, 1978, 1979; Ruina, 1983; Blanpied et al., 1995, 1998; Marone, 1998]. In the situations with constant effective normal stress $\bar{\sigma}$, the shear strength $\mathcal{T}$ is often expressed as

$$
\begin{aligned}
\mathcal{T} & =\psi(\dot{\delta}, \theta) \\
& =\bar{\sigma}\left[f_{0}+a \ln \left(\frac{\dot{\delta}}{\dot{\delta}_{0}}\right)+b \ln \left(\frac{\dot{\delta}_{0} \theta}{L}\right)\right],
\end{aligned}
$$

where $a>0$ and $b$ are RS constitutive parameters with magnitudes of the order of $0.01, \delta$ is the magnitude of slip velocity, $f_{0}$ is a reference friction coefficient corresponding to a reference slip velocity $\delta_{0}, \theta$ is a state variable which is typically interpreted as the average age of the population of contacts between two surfaces, and $L$ is the characteristic slip for state evolution [Dieterich, 1978, 1979; Rice and Ruina, 1983; Ruina, 1983; Dieterich and Kilgore, 1994]. Two types of state-variable evolution laws are commonly used in modeling:

$$
\begin{gathered}
\frac{d \theta}{d t}=1-\frac{\dot{\delta} \theta}{L} \quad \text { (aging law), } \\
\frac{d \theta}{d t}=-\frac{\dot{\delta} \theta}{L} \ln \left(\frac{\dot{\delta} \theta}{L}\right) \quad \text { (slip law). }
\end{gathered}
$$

[11] The parameter combination $a-b<0$ corresponds to steady state velocity-weakening friction and can lead to unstable slip, whereas $a-b>0$ corresponds to steady state velocity strengthening and leads to stable sliding [Rice and Ruina, 1983; Ruina, 1983]. Throughout this article, we omit the words "steady state" and simply refer to velocity weakening/strengthening.

[12] In expression (12), shear frictional strength $\mathcal{T}$ is undefined for slip velocities $\dot{\delta}=0$, which is unphysical. To regularize (12) near $\dot{\delta}=0$, we follow the approach of Rice and Ben-Zion [1996], Ben-Zion and Rice [1997], and Lapusta et al. [2000] in using a thermally activated creep model of the direct effect term $a \ln \left(\dot{\delta} / \dot{\delta}_{0}\right)$ to obtain

$$
\begin{aligned}
\mathcal{T} & =\psi(\dot{\delta}, \theta) \\
& =a \bar{\sigma} \operatorname{arcsinh}\left[\frac{\dot{\delta}}{2 \dot{\delta}_{0}} \exp \left(\frac{f_{0}+b \ln \left(\dot{\delta}_{0} \theta / L\right)}{a}\right)\right] .
\end{aligned}
$$

This regularization is used in our simulations. It produces a negligible change from (12) in the range of slip velocities explored by laboratory experiments; the difference in $\dot{\delta}$ at $\dot{\delta} \sim \dot{\delta}_{0}$ is of the order of $\exp \left(-2 f_{0} / a\right)$ or less, and the typical value of $f_{0} / a$ in this study is 75 .

\subsection{Updating Scheme: Advancing One Evolution Time Step}

[13] We have developed an updating scheme, based upon the explicit Newmark method described in section 2.1, appropriate for the RS fault boundary condition. Here, we 
discuss how values of field variables are updated over one evolution time step. Suppose that the discretized values of particle displacement $\mathbf{u}$, particle velocity $\dot{\mathbf{u}}$, and particle acceleration field $\ddot{\mathbf{u}}$ are known at the nth time step. To find the values of the field variables at the $(n+1)$ th time step, we perform the following steps.

[14] 1. Update the values of displacements, based on the known values at the $n$th time step:

$$
\mathbf{u}_{n+1}=\mathbf{u}_{n}+\Delta t \dot{\mathbf{u}}_{n}+\frac{1}{2}(\Delta t)^{2} \ddot{\mathbf{u}}_{n} .
$$

[15] 2. Perform the partial update of the particle velocity field in (4) by computing

$$
\dot{\mathbf{u}}_{n+1}^{*}=\dot{\mathbf{u}}_{n}+\frac{1}{2} \Delta t \ddot{\mathbf{u}}_{n}
$$

[16] 3. Compute the "stick" traction in (11):

$$
\begin{aligned}
& \dot{\mathbf{u}}_{n+1}^{\text {free }}=\dot{\mathbf{u}}_{n+1}^{*}-\frac{\Delta t}{2}\left(\mathbf{M}^{-1} \mathbf{K} \mathbf{u}_{n+1}\right) \\
& \tilde{\mathbf{T}}_{n+1}=\tau_{o}+\mathbf{Z} \dot{\delta}_{n+1}^{\text {free }} .
\end{aligned}
$$

[17] 4. Determine the first prediction of the state variable, $\theta_{n+1}^{*}$. By integrating the evolution law (13) or (14) with the constant magnitude $\dot{\delta}_{n}$ of slip velocity $\dot{\delta}_{n}=\mathbf{u}_{n}^{+}-\mathbf{u}_{n}^{-}$during the time step, we obtain

$$
\theta_{n+1}^{*}=\theta_{n} \exp \left(-\frac{\dot{\delta}_{n} \Delta t}{L}\right)+\frac{L}{\dot{\delta}_{n}}\left(1-\exp \left(-\frac{\dot{\delta}_{n} \Delta t}{L}\right)\right)
$$

for the aging law, and

$$
\theta_{n+1}^{*}=\frac{L}{\dot{\delta}_{n}}\left(\frac{\dot{\delta}_{n} \theta_{n}}{L}\right)^{\exp \left(-\dot{\delta}_{n} \Delta t / L\right)}
$$

for the slip law. This approach for updating the state variable is different from the one by Lapusta et al. [2000]. We compare the state-variable updating schemes in section 3.

[18] 5. Find the first prediction of slip velocity, $\dot{\delta}_{n+1}^{*}$, by equating the magnitude of shear stress in (11) and strength in (15). The directions of shear traction vector $\mathbf{T}_{n+1}$ and slip velocity vector $\dot{\boldsymbol{\delta}}_{n+1}$ have to coincide. From (11), the stick traction $\tilde{\mathbf{T}}_{n+1}$ has the same direction because the fault impedance matrix $\mathbf{Z}$ is isotropic (Appendix A). By projecting (11) onto that direction and equating the shear stress magnitude with frictional strength, we obtain the following relation:

$$
T_{n+1}^{*}=\tilde{T}_{n+1}-Z_{\mathrm{x}} \dot{\delta}_{n+1}^{*}=\psi\left(\dot{\delta}_{n+1}^{*}, \theta_{n+1}^{*}\right)
$$

where $T$ and $\tilde{T}$ denote the magnitudes of $\mathbf{T}$ and $\tilde{\mathbf{T}}$, respectively. We find $\dot{\delta}_{n+1}^{*}$ using the Newton-Raphson search with $\dot{\delta}_{n}$ as the first guess. Once $\dot{\delta}_{n+1}^{*}$ are obtained, the traction $T_{n+1}^{*}$ can be readily found.

[19] 6. Calculate the final prediction of state variable, $\theta_{n+1}^{* *}$, at the $(n+1)$ th time step by replacing $\dot{\delta}_{n}$ in equation (19) or (20) with $\left(\dot{\delta}_{n}+\dot{\delta}_{n+1}^{*}\right) / 2$.
[20] 7. Find the final predictions $\dot{\delta}_{n+1}^{* *}$ and $T_{n+1}^{* *}$ by repeating step 5 with $\theta_{n+1}^{* *}$ instead of $\theta_{n+1}^{*}$.

[21] 8. Declare the value of $T_{n+1}$ to be equal to the predictions with the superscript double asterisks. Using the directional cosines constructed from the components of $\tilde{\mathbf{T}}_{n+1}$, we obtain the components of $\mathbf{T}_{n+1}$ and relative traction $\tau_{n+1}$.

[22] 9. Solve for acceleration of the entire medium:

$$
\ddot{\mathbf{u}}_{n+1}=\mathbf{M}^{-1}\left[-\mathbf{K} \mathbf{u}_{n+1}+\mathbf{B} \tau_{n+1}\right] .
$$

[23] 10. Complete the update of $\dot{\mathbf{u}}_{n+1}$ by adding the term containing $\ddot{\mathbf{u}}_{n+1}$ :

$$
\dot{\mathbf{u}}_{n+1}=\dot{\mathbf{u}}_{n+1}^{*}+\frac{1}{2} \Delta t \ddot{\mathbf{u}}_{n+1}
$$

[24] This scheme includes two iterations for the update of the state variable. Its accuracy, for a given space grid, is comparable to that of BIM, as discussed in section 3. Note that if the second iteration in the state-variable update is omitted, the accuracy significantly decreases in comparison to BIM which also includes two iterations for the update of the state variable.

\section{Comparison of Numerical Results Obtained With 2-D SEM and 2-D BIM}

\subsection{Two-Dimensional Antiplane Problem and Comparison Criteria}

[25] To assess the accuracy of numerical results based on the developed SEM approach, we have conducted detailed comparison of simulation results obtained using SEM and BIM. For simplicity and efficiency, we set up an antiplane (2-D) test problem. The BIM model used for comparison is based on the BIM spectral formulation of Lapusta et al. [2000]. Figure 2 illustrates the geometry of the antiplane SEM and BIM models. In SEM, a $15 \mathrm{~km}$ by $45 \mathrm{~km}$ rectangular domain is used, with four boundaries. The SEM model domain is large enough to avoid any wave reflections from the boundaries during the simulated time. The domain is replicated using periodic boundary conditions on both sides of the domain (Figure 2). The fault boundary obeys rate and state (RS) friction with the aging law of state variable evolution. By symmetry consideration, the medium across the fault boundary has equal and opposite motion. In the analogous BIM model, wave propagation is analytically accounted for by boundary integral expressions. The fault in the BIM model is repeated periodically, as in the SEM model.

[26] The parameters used in the simulations are listed in Table 1, and the distribution of initial shear stress on the fault is shown in Figure 3a. We make our test problem similar to the SCEC code validation of dynamic rupture [Harris et al., 2004] and the study of Day et al. [2005], in terms of bulk properties and cohesive zone properties, but we use RS friction and consider a 2-D scenario. Within the $3-\mathrm{km}$ nucleation region, we use an integer number of spectral elements for SEM and an odd number of computational cells for BIM. We select the polynomial degree to be 4 in SEM throughout the comparison. At the nucleation 
2D SEM

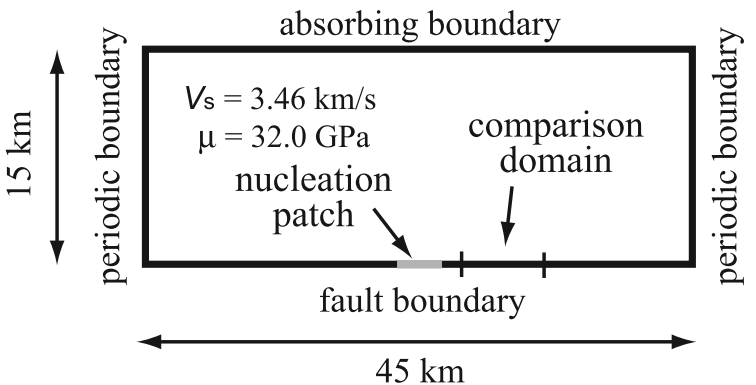

2D BIM

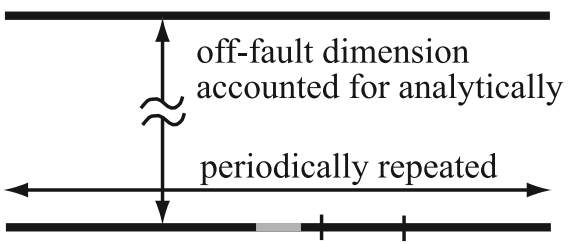

fault boundary

Figure 2. A cartoon illustrating the antiplane test problem for (left) 2-D SEM and (right) 2-D BIM. By symmetry consideration, the medium across the fault boundary in both models has equal and opposite motion.

patch in the center of the fault, slip velocity increases abruptly because of the difference between the initial shear stress imposed and initial shear strength given by the RS friction. (Note that the initial bulk particle velocity is uniform and equal to half of initial slip velocity on the fault outside the nucleation patch.) The resulting dynamic rupture propagates bilaterally from the nucleation patch. On the RS fault, friction strength is not known in advance as it depends on the current values of slip velocity and state variable. The RS parameters we use (Table 1) result in effective slip weakening similar to the LSW friction of the SCEC code validation (Figure $3 b$ ).

[27] To quantify differences between solutions, we use a quantity analogous to the rupture arrival time. For problems with spontaneous dynamic rupture, the rupture arrival time has been shown to be a sensitive indicator of numerical precision that reflects the nonlinearity of the problem [Day et al., 2005]. In the SCEC code validation [Harris et al., 2004] and the study of Day et al. [2005], rupture arrival time was defined as the time when slip velocity first exceeds $1 \mathrm{~mm} / \mathrm{s}$. In this study, we use the arrival time of peak slip velocity (PSV), an analogous quantity. This quantity is more convenient for RS faults as it does not require choosing a particular value of slip velocity threshold and can be used in a wider context, for example, for comparing rupture arrival times in velocity-strengthening regions, which may not achieve slip velocity of $1 \mathrm{~mm} / \mathrm{s}$. To compare two solutions, we use root-mean-square (RMS) difference of PSV arrival times, interpolated with the spacing of $0.1 \mathrm{~km}$, over the fault region $\Omega$ given by $3.0 \mathrm{~km} \leq x \leq$ $9.0 \mathrm{~km}$.
[28] The numerical accuracy critically depends on the ratio $N_{c}=\Lambda / \Delta x$ or the number of fault node points (with average spacing $\Delta x$ ) within the cohesive (or slip weakening) zone size $\Lambda$ [e.g., Day et al., 2005]. We indicate the average cohesive zone resolution $\bar{N}_{c}$ over the fault domain $\Omega$ in the comparisons that follow. Note that the value of $\Lambda$ for the quasi-stationary crack [e.g., Rice, 1980; Day et al., 2005 ] is $0.62 \mathrm{~km}$ for this problem. By comparing simulations with different spatial and temporal resolution, we find, consistently with Day et al. [2005], that locations of the highest error correspond to fault regions far from the nucleation patch, because the cohesive zone size gradually shrinks as rupture accelerates along a homogeneously prestressed fault. Hence differences in PSV arrival time at a point close to the end of the ruptured region (e.g., $x=9 \mathrm{~km}$ ) would be a more sensitive indicator of the accumulated error than the RMS difference over the domain. However, RMS values are more indicative of the overall error and help avoid the error scatter through averaging.

\subsection{Convergence of SEM and BIM Solutions With Grid Reduction}

[29] SEM and BIM simulations with high resolution (i.e., large $\bar{N}_{c}$ ) result in virtually identical solutions, in the sense that the difference in PSV arrival times and in peak slip velocities is negligible compared to their absolute values. As an example, consider our highest-resolution SEM and BIM simulations, which result in $\bar{N}_{c}$ of about 22. (The average node spacing of the highest-resolution simulations is $0.0093 \mathrm{~km}$ in SEM and $0.0074 \mathrm{~km}$ in BIM). At one of the most computationally demanding locations, $x=9 \mathrm{~km}$, the

Table 1. Friction-Related Parameters Used in 2-D and 3-D Simulations

\begin{tabular}{lccc}
\hline \multicolumn{1}{c}{ Parameter } & Symbol & Value in Two Dimensions & Value in Three Dimensions \\
\hline Reference slip velocity & $\dot{\delta}_{0}$ & $10^{-6} \mathrm{~m} / \mathrm{s}$ & $10^{-6} \mathrm{~m} / \mathrm{s}$ \\
Reference friction coefficient & $f_{0}$ & 0.60 & 0.60 \\
Characteristic slip distance & $L$ & $0.0370 \mathrm{~m}$ & $0.0135 \mathrm{~m}$ \\
Effective normal stress & $\bar{\sigma}$ & $120.0 \mathrm{MPa}$ & $80.0 \mathrm{MPa}^{\mathrm{a}}$ \\
Initial slip velocity & $\dot{\delta}_{\text {in }}$ & $10^{-3} \mathrm{~m} / \mathrm{s}$ & $10^{-12} \mathrm{~m} / \mathrm{s}$ \\
Initial state variable & $\theta_{\text {in }}$ & $92.7 \mathrm{~s}$ & 34.38 years \\
Constitutive parameter $a$ & $a$ & 0.0125 & $0.0080^{\mathrm{b}}$ \\
Constitutive parameter $b$ & $b$ & 0.0172 & $0.0120^{\mathrm{a}}$ \\
\hline
\end{tabular}

${ }^{\mathrm{a}}$ The values in the region of constant $(a-b)$ in the velocity-weakening area (Figure 8) are given.

${ }^{\mathrm{b}}$ The value within the rupture domain (Figure 3 ) is given. 
(A)

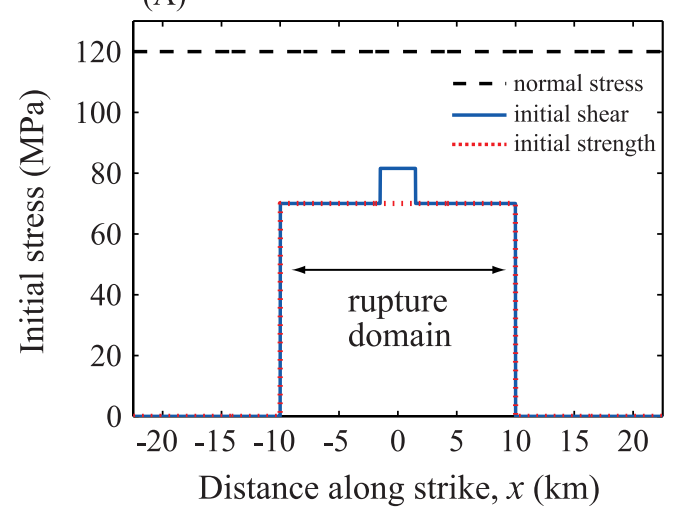

(B)

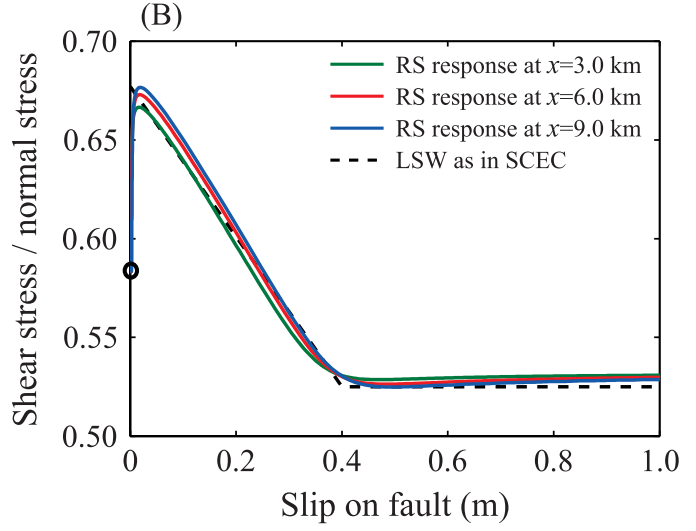

Figure 3. (a) Initial stress distribution, similar to that of the SCEC code validation. (b) The effective slip dependence of rate and state (RS) friction. With the parameters listed in Table 1, the resulting effective slip dependence of the RS interface (solid lines) over the comparison domain matches very closely the LSW friction in the SCEC validation problem (dashed line). The open circle corresponds to the coefficient of friction associated with the initial strength of the comparison domain.

difference in the PSV arrival times is $3 \times 10^{-4} \mathrm{~s}$ or $0.01 \%$ of the PSV arrival time and the difference in peak velocity is $5 \times 10^{-3} \mathrm{~m} / \mathrm{s}$ or $0.05 \%$ of the peak velocity. The nearly identical slip velocity histories are shown in Figure $4 \mathrm{a}$. Note that the slip velocity history has a pulse-like shape due to the stopping phase arriving from the rupture arrest at $x=$ $10 \mathrm{~km}$.

[30] The convergence of SEM and BIM solutions with grid reduction is shown in Figure $4 \mathrm{~b}$. The quantity plotted is the root-mean-square (RMS) difference of PSV arrival times relative to the highest-resolution runs; the result is expressed as a percentage of the RMS arrival time of the highestresolution run. To determine PSV arrival times, we use interpolation of near-peak slip velocity history by piecewise cubic splines with time intervals much smaller than the time step of the highest-resolution simulations. Open circles in Figure 4b show RMS difference of PSV arrival times as a function of average node spacing for SEM calculations, using the SEM highest-resolution simulation as the reference. Open triangles show the same quantity for BIM, using the BIM highest-resolution simulation as the reference. The differences in PSV arrival times approximately follow a power law in the average node spacing, with the estimated exponents of 1.85 for SEM and 1.93 for BIM. Note that the convergence exponent is similar for SEM and BIM. The estimated cohesive zone size $\Lambda$ averaged over the comparison domain is $0.21 \mathrm{~km}$, and the numerical accuracy depends on the cohesive zone resolution $\bar{N}_{c}$, consistently with the study by Day et al. [2005].

[31] The results show that the highest-resolution solutions, convergence rates, and errors for both SEM and BIM are nearly identical, validating our SEM algorithm.

\subsection{Evaluation of State-Variable Updating Schemes}

[32] The results in section 3.2 are obtained using the state-variable updating scheme (19). An alternative way of updating the state variable is to use the following relations in the updating scheme (section 2.3):

$$
\begin{aligned}
& \theta_{n+1}^{*}=\theta_{n}+\Delta t \dot{\theta}_{n} \text { at step } 4, \\
& \theta_{n+1}^{* *}=\theta_{n}+\frac{\Delta t}{2}\left(\dot{\theta}_{n}+\dot{\theta}_{n+1}^{*}\right) \text { at step } 6,
\end{aligned}
$$

where $\dot{\theta}_{n}$ is obtained from the state-variable evolution laws (13) or (14) with $\dot{\delta}=\dot{\delta}_{n}$ and $\theta=\theta_{n}$. This approach was used by Lapusta et al. [2000]. Since $\theta^{*}{ }_{n+1}$ in (24) is the firstorder expansion of (19) and (20) with respect to $\Delta t$ for a constant slip velocity, one would expect the state-variable updating scheme (24) to be less accurate. We verify this expectation by numerical simulations (Figures $4 \mathrm{~b}$ and $4 \mathrm{c}$ ). While the two updating schemes are comparable for fine discretizations $\left(N_{c} \gtrsim 2\right)$, the errors for the updating scheme (24) are higher in the case of coarser discretizations $\left(N_{c} \lesssim 2\right)$, for both SEM and BIM. The error difference for coarser discretizations may be important in 3-D simulations, which tend to require marginal discretizations due to their demand on computational resources.

\subsection{Comparison of Simulations With Linear Slip- Weakening and Rate and State Friction}

[33] LSW friction laws are widely used to simulate dynamic rupture. Several dynamic rupture codes for LSW faults have been compared in the SCEC code validation [Harris et al., 2004]. LSW laws incorporate discontinuities in derivatives, such as the abrupt change from weakening to a constant dynamic friction level. RS laws, on the contrary, are smooth. Hence, it is reasonable to hypothesize that RS laws would lead to simulations with better numerical accuracy than LSW laws. Since the aging form of RS laws can match the overall shape of LSW laws quite well, we can use our simulations to test that conjecture. We use the same LSW friction as in the SCEC code comparison, and the parameters of RS friction are chosen to match that LSW friction during dynamic rupture (Figure 3).

[34] Figure 5a shows that the two laws result in SEM simulations with comparable accuracy. However, there are important differences. For the node spacing larger than $\sim 0.1 \mathrm{~km}$, the errors of solutions with LSW friction are 33\% higher on average than those with the aging law of RS friction. Hence, as expected, RS friction results in smaller errors for the same node spacing. Even though the computational time for the same node spacing is larger for RS 
friction than LSW friction, one can obtain the same accuracy using a coarser node spacing in the calculations with $\mathrm{RS}$ friction, actually reducing the computational time in comparison with LSW. To illustrate this point, we consider RMS difference in PSV arrival time over the domain $8.5 \mathrm{~km} \leq$ $x \leq 9.0 \mathrm{~km}$, where the errors would be expected to be higher than for smaller $x$ (Figure 5a inset). LSW simulation with $\Delta x=0.107 \mathrm{~km}$ and RS simulation with $\Delta x=0.125 \mathrm{~km}$

(A)
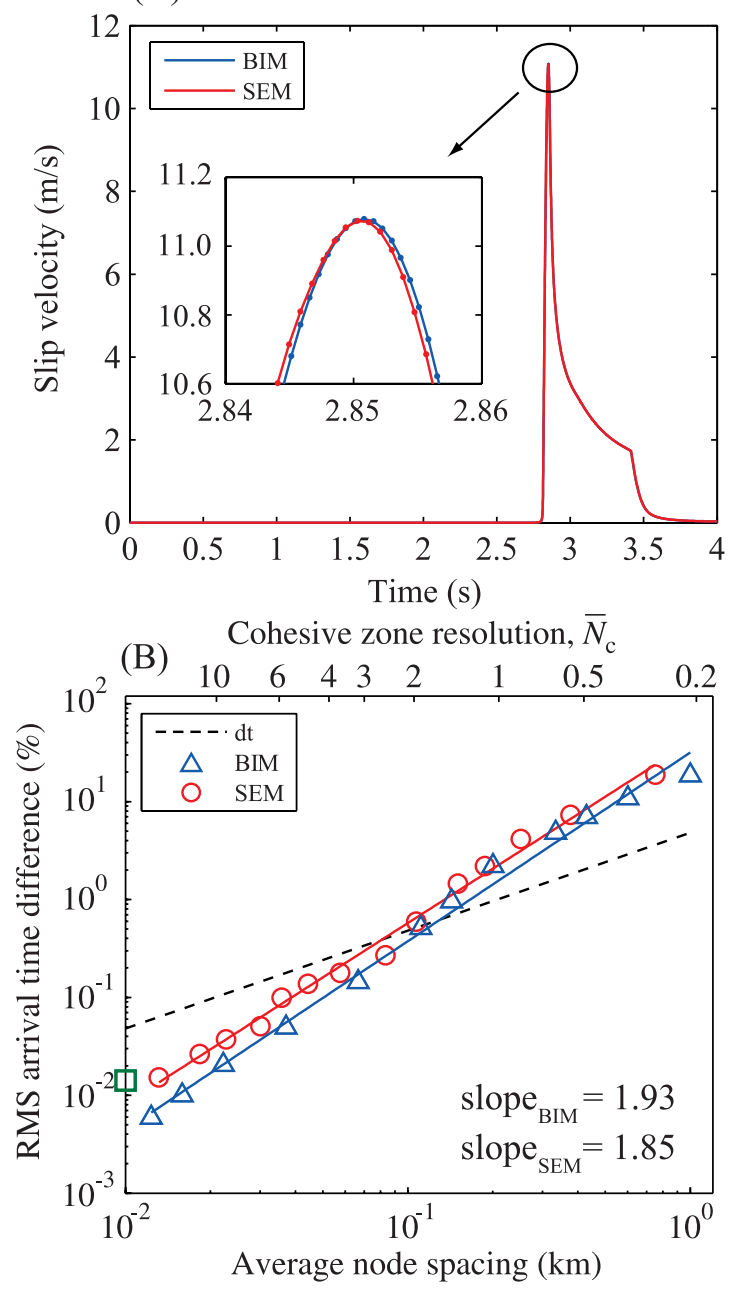

(C)

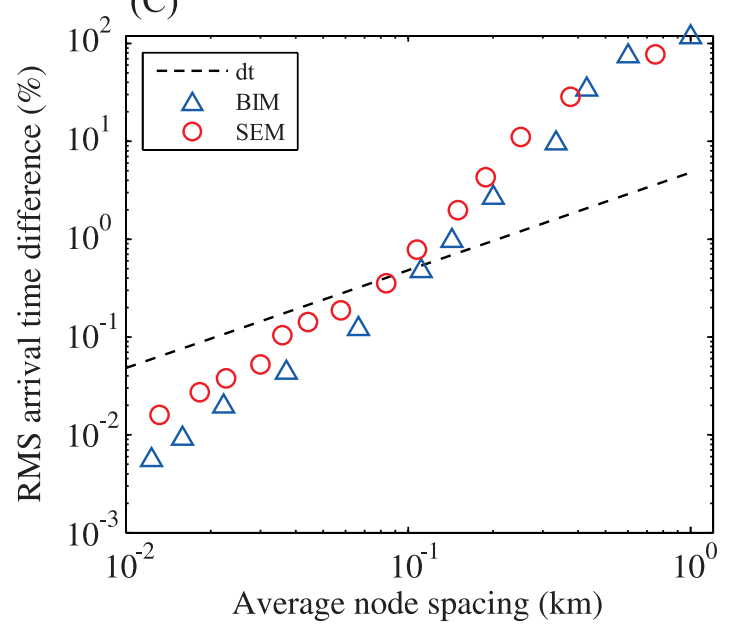

have comparable error of about $1 \%$. For the node spacing of $0.107 \mathrm{~km}$, the CPU time is 1.35 times longer in the calculation with RS friction than in that with LSW friction. However, the CPU time and memory in the RS calculation with the node spacing of $0.107 \mathrm{~km}$ are 1.53 and 1.36 times larger, respectively, than the one with $0.125 \mathrm{~km}$. Hence, RS calculation with $\Delta x=0.125 \mathrm{~km}$ takes $12 \%$ smaller CPU time and 36\% smaller memory than the LSW calculation with $\Delta x=0.107 \mathrm{~km}$ that has comparable accuracy. This illustrates that the computational cost with respect to a given accuracy of a solution is smaller in calculations with RS friction than in those with LSW friction. Note that the differences in CPU time and memory become even larger for 3-D computations, as the differences increase with the number of the node points.

[35] In addition, numerical oscillations, caused by discontinuities of derivatives in LSW friction, increase numerical noise and prevent further reduction of errors for finer discretizations. Such numerical oscillations are appreciably smaller in simulations with RS friction than in those with LSW friction, as Figure 5b shows for the node spacing of $0.036 \mathrm{~km}$. The oscillatory slip velocity near the peak in the LSW simulation results in much higher errors for finer discretizations (Figure 5b). Note that numerical damping in the form of Kelvin-Voigt viscosity is often used to suppress numerical noise for the calculations with LSW friction [e.g., Day et al. 2005]. Our experience with using numerical damping indicates that it also results in slower rupture speeds (longer PSV arrival times).

[36] We quantify the degree of smoothness of the solutions obtained using the two friction laws by comparing the spectra of slip velocity (Figure 5c). The spectra differ for high frequencies $(f>20 \mathrm{~Hz})$. While that frequency range cannot be resolved by inversions of seismological data and it is beyond the frequency band relevant for most engineering applications, it reveals important differences in the behavior of the two solutions. The decay of high frequencies $(20 \mathrm{~Hz} \lesssim f \lesssim 100 \mathrm{~Hz}$ ) for the simulation with $\mathrm{RS}$

Figure 4. (a) Slip velocity histories at $x=9.0 \mathrm{~km}$, for the highest-resolution simulations of SEM and BIM. The slip velocity histories are nearly identical as quantified in the text. (b) Differences in arrival time of peak slip velocity (PSV), relative to the highest-resolution runs, shown as a function of average node spacing. Differences are given as RMS averages over the domain $3.0 \mathrm{~km} \leq x \leq 9.0 \mathrm{~km}$ normalized by the RMS arrival time of the highestresolution runs. Circles are SEM solutions, relative to the highest-resolution run of SEM, and triangles are BIM solutions, relative to the highest-resolution run of BIM. The square on the vertical axis corresponds to the difference between the SEM and BIM highest-resolution runs. The dashed line shows the dependence of time step $\Delta t$, set to be equal for both SEM and BIM, on average node spacing, normalized by the RMS arrival time of the highestresolution runs. The top axis gives the average cohesive zone resolution $\bar{N}_{c}$. (c) Errors of BIM and SEM solutions with the alternative state-variable updating scheme (24). Comparison with Figure $4 \mathrm{~b}$ shows that for discretizations above $10^{-1} \mathrm{~km}$, the errors of both SEM and BIM solutions are higher for this updating scheme. 
friction is approximately proportional to $f^{-5 / 2}$, which is faster than the $f^{-3 / 2}$ decay for the simulation with LSW friction. Hence slip velocity at the onset of sliding for RS and LSW friction are proportional to $t^{3 / 2}$ and $t^{1 / 2}$, respectively, and the corresponding slip acceleration is proportional to $t^{1 / 2}$ and $t^{-1 / 2}$. This behavior of the numerical solutions indicates that in the corresponding continuum solutions for LSW, slip acceleration at the onset of sliding is infinite, consistently with the finding of Ida [1973], and abruptly jumps from zero, whereas slip acceleration for RS

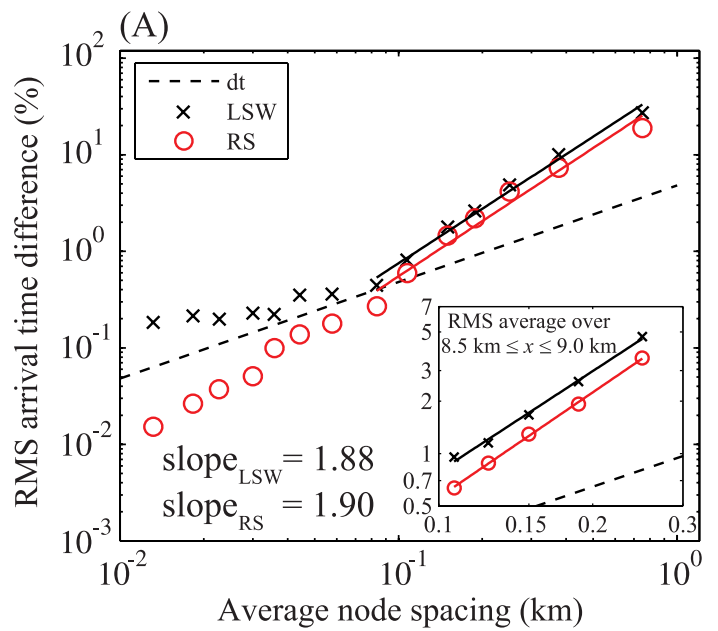

(B)

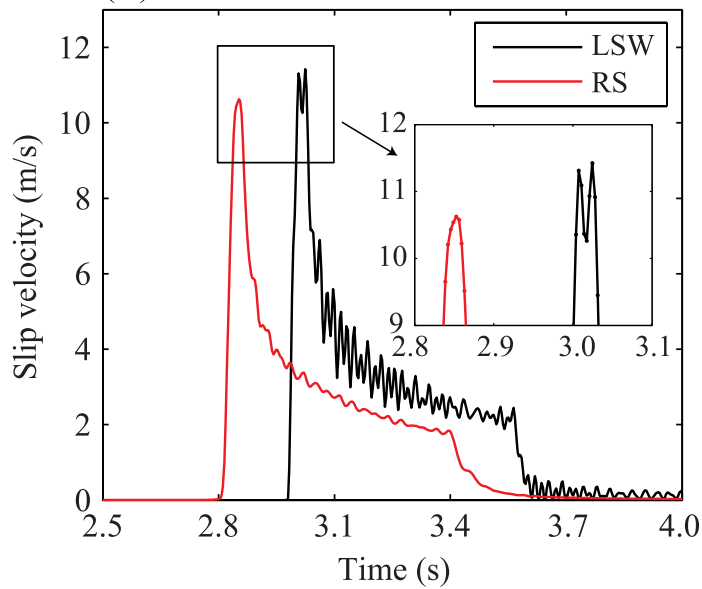

(C)

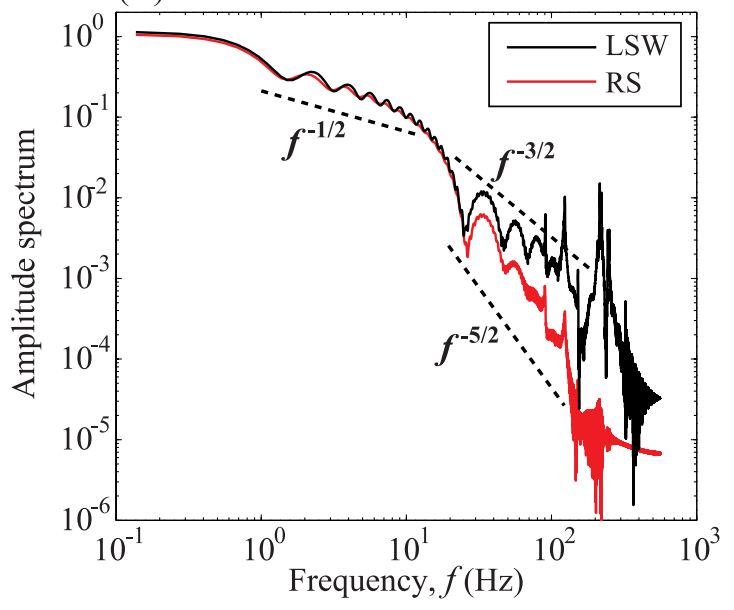

friction is finite and smoothly changes from zero. The discontinuity in the continuum solution with LSW friction limits the quality of the corresponding numerical solutions. That is why the model with RS friction results in numerical solutions with smaller oscillations and has better convergence than that with LSW friction (Figures $5 \mathrm{a}$ and $5 \mathrm{~b}$ ).

[37] We conclude that for a given accuracy of solutions, the aging form of RS law leads to more stable and accurate simulations than LSW law, while producing savings in computational time and memory.

\subsection{Simulations With the Slip Law of State-Variable Evolution}

[38] In addition to the aging law considered so far, other formulations of the state-variable evolution have been proposed, including the slip law, the composite law, and laws with more than one state variable [Ruina, 1983; Rice and Ruina, 1983; Gu et al., 1984; Kato and Tullis, 2001]. Several recent nucleation studies [Kato and Tullis, 2001; Rubin and Ampuero, 2005; Ampuero and Rubin, 2008] found notable differences between models with different state-variable evolution laws. Which formulations most adequately represent laboratory experiments is a question of active current research [e.g., Bayart et al., 2006].

[39] Figure 6a shows that the errors of SEM solutions with the slip law are much higher than those with the aging law, for the same parameters. In comparison with the aging law, the slip law requires much higher numerical resolution to establish the same order of accuracy. This is because the effective slip-weakening rate (i.e., the rate of stress decrease with slip) is variable for the slip law, with the maximum effective slip-weakening rate larger in simulations with the slip law than with the aging law for the same RS parameters (Figure 6b) by a logarithmic factor of peak slip velocity [Ampuero and Rubin, 2008]. In our test problem, when the value of the characteristic slip $L$ in the slip law is increased eight times, the maximum effective slip-weakening rate becomes approximately equal for both laws in this particular model, and the errors in simulations with the slip law (Figure 6a) become comparable to the errors in the simu-

Figure 5. (a) Comparison of errors for SEM solutions with LSW and RS friction. The errors for LSW are consistently higher. The inset shows comparison of RMS arrival time difference over the domain $8.5 \mathrm{~km} \leq x \leq 9.0 \mathrm{~km}$. (b) Slip velocity histories at $x=9.0 \mathrm{~km}$ for the simulations with the average node spacing of $0.036 \mathrm{~km}$. The simulation with LSW has much larger numerical oscillations. Note that the visible time difference between rupture arrivals for the two cases arises because the two simulations are not identical in terms of the friction law, although they are very similar in terms of slip-weakening behavior. The faster rupture arrival in the simulation with RS friction indicates a larger average rupture speed and hence a more challenging calculation. (c) Amplitude spectra of slip velocity at $x=9.0 \mathrm{~km}$ for the highest-resolution SEM simulations with LSW and RS friction. The faster decay of the high-frequency $(f \gtrsim 20 \mathrm{~Hz})$ part of the spectra explains the superior convergence behavior and numerical stability of simulations with RS friction as discussed in the text. 
(A)

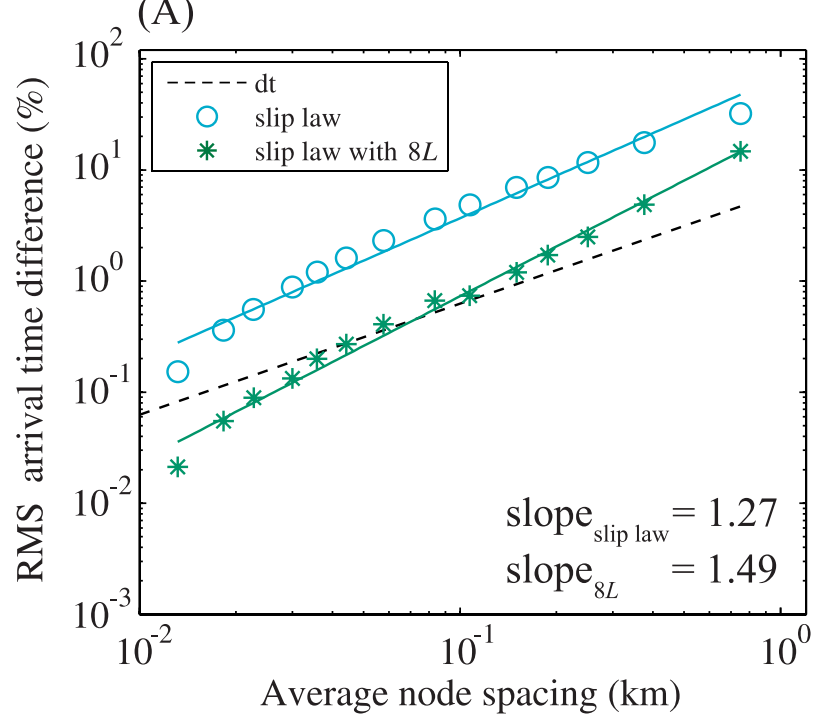

(B)

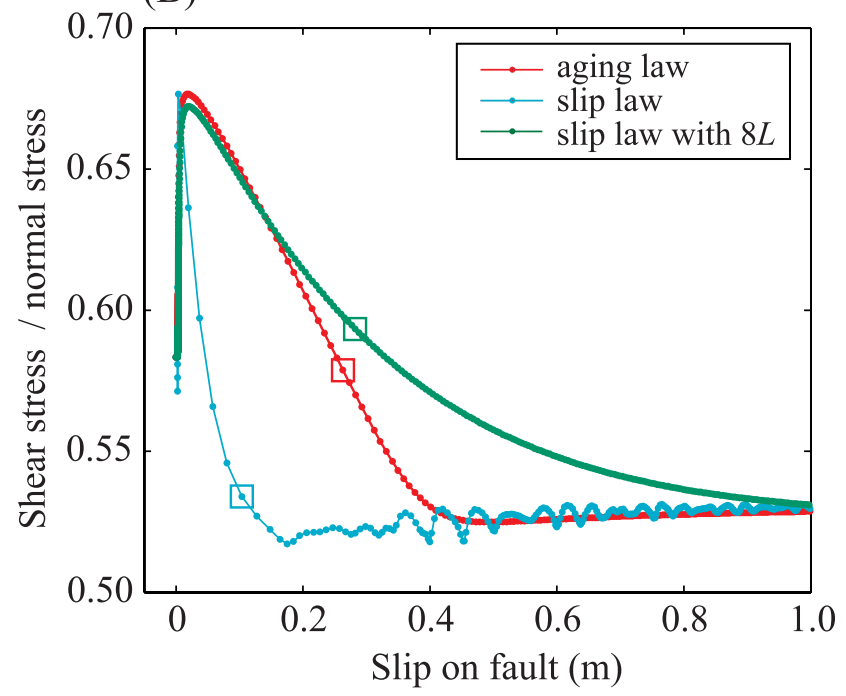

Figure 6. (a) Errors for SEM solutions with the slip law of state-variable evolution. Blue circles correspond to the errors for SEM calculations with the parameters of Table 1. Green stars correspond to the errors for SEM calculations with eight times larger characteristic slip $L$. The overall errors for the slip law with eight times larger $L$ is comparable to that of the aging law shown in Figure 4b. (b) Effective slip dependence of the friction coefficient at $x=9.0 \mathrm{~km}$ for the highest-resolution simulations with the aging law, the slip law, and the slip law with eight times larger $L$. The squares show the values of stress and slip at the PSV arrival time at $x=9.0 \mathrm{~km}$.

lations with the aging law (Figure $4 b$ ). These results suggest that errors are controlled by the resolution of the maximum effective slip-weakening rate. Note that this is consistent with the notion that the resolution of cohesive zone controls errors for calculations with LSW friction and the aging law of RS friction. In LSW friction, effective slip-weakening rate at the rupture front is constant, and the cohesive zone size reflects that rate. The aging law results in slipdependent behavior close to that of LSW friction (Figure 6b).

\section{Effect of Velocity-Strengthening Fault Friction at Shallow Depths on Dynamic Rupture}

[40] We use the SEM for rate and state faults developed in section 2 and validated in section 3 to investigate the effect of different fault rheologies on dynamic rupture in a 3-D fault model. The fault is governed by the aging form of RS friction, with relatively uniform prestress. We consider two dynamic rupture scenarios on a vertical strike-slip fault embedded into an elastic half-space: case 1 with velocityweakening friction extending up to the free surface, and case 2 with a shallow $3-\mathrm{km}$ velocity-strengthening region next to the free surface (Figure 7). Case 2 is motivated by the inferred existence of a velocity-strengthening fault rheology at shallow depths as described in section 1 . Absorbing conditions [Clayton and Engquist, 1977] are used on all boundaries of the SEM model except the free surface and the fault boundary, to simulate a semi-infinite elastic half-space.

[41] Figures 7 and 8 illustrate parameters and initial conditions for the SEM model. The effective normal stress, $\bar{\sigma}=\min [1.0+16.2 z, 80.0] \mathrm{MPa}$, where $z$ is in kilometers, increases with depth because of the difference of overburden minus hydrostatic pore pressure and becomes constant $(80.0 \mathrm{MPa})$ at depths larger than $4.9 \mathrm{~km}$, because of the assumption that fluid overpressure prevents further increase of $\bar{\sigma}$ with depth [Rice, 1993; Ben-Zion and Rice, 1997]. We compute the initial stress distribution in Figure 8a on the basis of (12) by assuming that initial slip velocity $\dot{\delta}_{\text {in }}=$ $10^{-12} \mathrm{~m} / \mathrm{s}$ and initial state variable $\theta_{\text {in }}=34.38$ years are constant throughout the fault (Table 1). The initial stress distribution at shallow depths are slightly different in cases 1 and 2 owing to the difference in the friction parameters $a$ and $b$.

[42] The medium is initially moving on the two sides of the fault with equal and opposite horizontal particle velocities of $\dot{\delta}_{\text {in }} / 2=5 \times 10^{-13} \mathrm{~m} / \mathrm{s}$, values much smaller than typical plate loading rates of $10^{-10}$ to $10^{-9} \mathrm{~m} / \mathrm{s}$. Starting at time $t=0$, dynamic rupture is initiated by imposing a rapid but smooth time-dependent variation of the horizontal shear traction in a circular patch [Rojas et al., 2007; E. Dunham, personal communication, 2003] (Appendix B). This initiation procedure results in fast but gradual variations in slip velocity, producing more numerically stable results for lower numerical resolutions in comparison to the more abrupt initiation procedure (in the form of an overstressed patch of higher initial stress) of section 2 . The difference between the two procedures is especially important in 3-D problems, which are expensive computationally.

[43] Dynamic ruptures in the two cases are compared in Figures 9-12. Each simulation has an average node spacing of $0.050 \mathrm{~km}$ and takes several hours on 100 to 200 processors on California Institute of Technology (Caltech) supercomputer, CITerra. Figure 9 gives snapshots of the strike-parallel component of slip velocity every $2 \mathrm{~s}$ for 


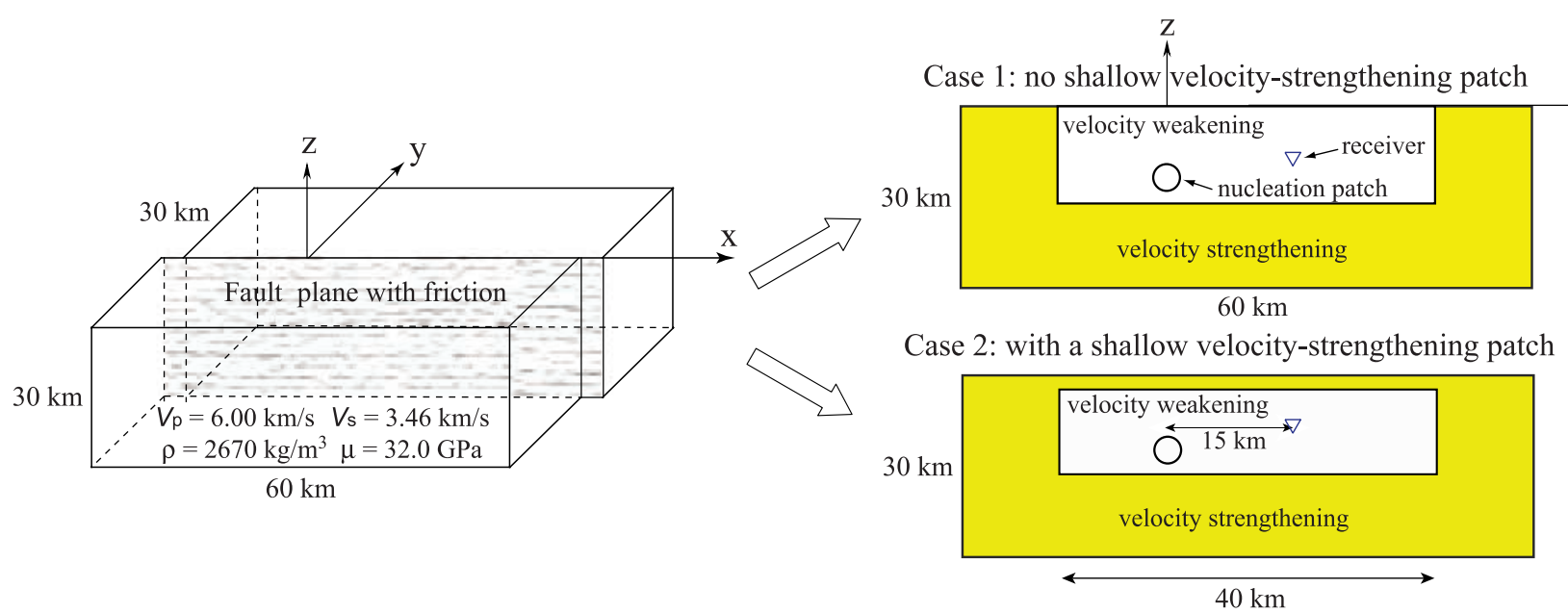

Figure 7. A 3-D model of a vertical strike-slip fault embedded into an elastic half-space. Two cases with different fault rheologies are considered, with and without shallow velocity-strengthening patch. At the horizontal transitions from velocity-weakening to velocity-strengthening properties $(x=-10 \mathrm{~km}$ and $x=30 \mathrm{~km}$ ), the value of $a$ stays constant, and the value of $(a-b)$ abruptly changes from -0.004 (velocity weakening) to 0.004 (velocity strengthening). The depth dependence of $a$ and $(a-b)$ within the region $-10 \mathrm{~km}<x<30 \mathrm{~km}$ is shown in Figure 8 .

case 1 (Figure 9, top) and case 2 (Figure 9, bottom). The rupture initiates at the nucleation patch and then spontaneously propagates until it encounters velocity-strengthening regions at the $15-\mathrm{km}$ depth and at lateral distances of -10 $\mathrm{km}, 30 \mathrm{~km}$, where slip gradually terminates. The resulting slip dependence of the RS simulation at the fault location $(x$, $z)=(15 \mathrm{~km},-7.5 \mathrm{~km})$ is shown in Figure $8 \mathrm{c}$.

\subsection{Suppression of Supershear Rupture Near the Free Surface}

[44] Significant differences between the two cases start to arise when the rupture reaches the velocity-strengthening patch next to the free surface. In the absence of the shallow velocity-strengthening patch (case 1), the rupture front next to the free surface propagates with a supershear speed (Figure 9). This supershear rupture propagation near the free surface has been observed in simulations with LSW faults [Aagaard et al., 2001; Day et al., 2008].

[45] In the presence of the shallow velocity-strengthening patch (case 2), the supershear rupture propagation near the free surface is suppressed (Figure 9). This is consistent with the fact that supershear rupture propagation near the free surface has not been commonly reported in large crustal earthquakes. Our results indicate that velocity-strengthening friction at shallow depths may account for, or at least contribute to, the lack of universal supershear rupture near the free surface. Other factors might contribute to suppression of supershear propagation at the free surface, such as a potential increase in breakdown work close to the free surface due to more distributed shear at low normal stresses or lower initial shear stress than assumed in this work.
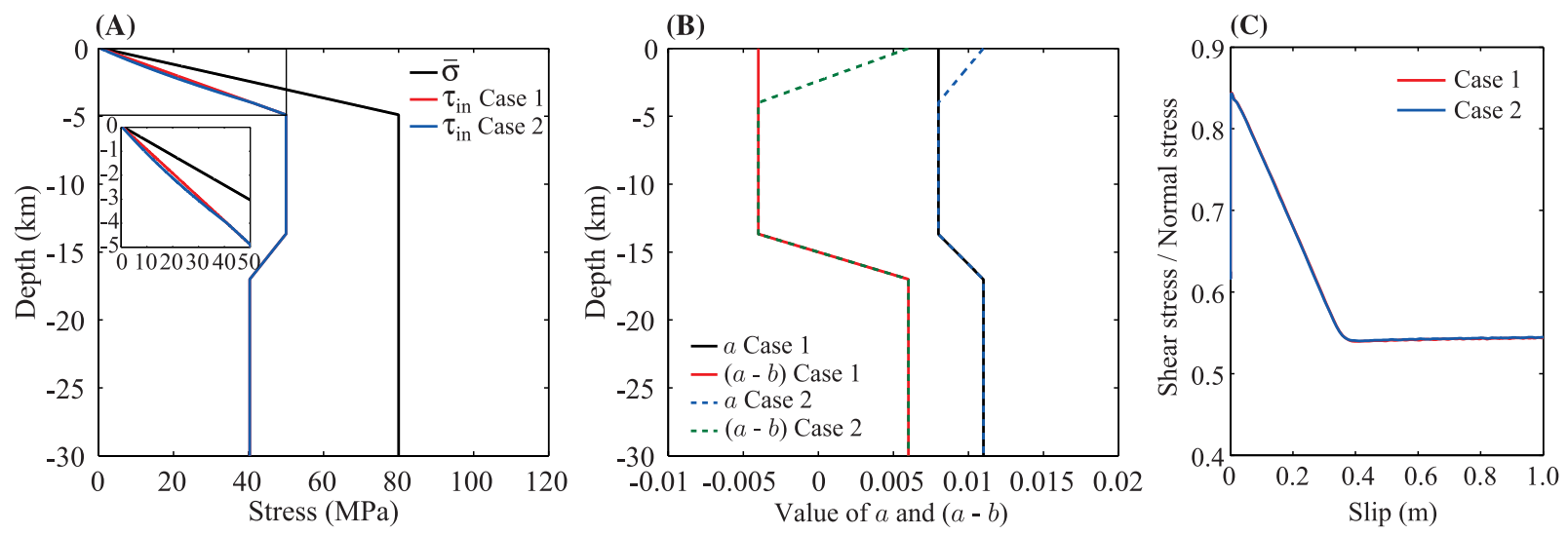

Figure 8. (a) Depth-variable distribution of effective normal stress and initial horizontal shear traction in cases 1 and 2 . The initial shear traction for $z \leq 5.0 \mathrm{~km}$ is slightly different in each case due to the difference in friction parameters $a$ and $b$. The initial shear strength is equal to the initial horizontal shear traction. (b) Depth-variable distribution of the parameters $(a-b)$ and $a$ within the region $-10 \mathrm{~km}<x<30 \mathrm{~km}$. (c) The resulting effective slip dependence of friction at the fault location $(x, z)=(15 \mathrm{~km},-7.5 \mathrm{~km})$. 
Case 1: No shallow velocity-strengthening patch
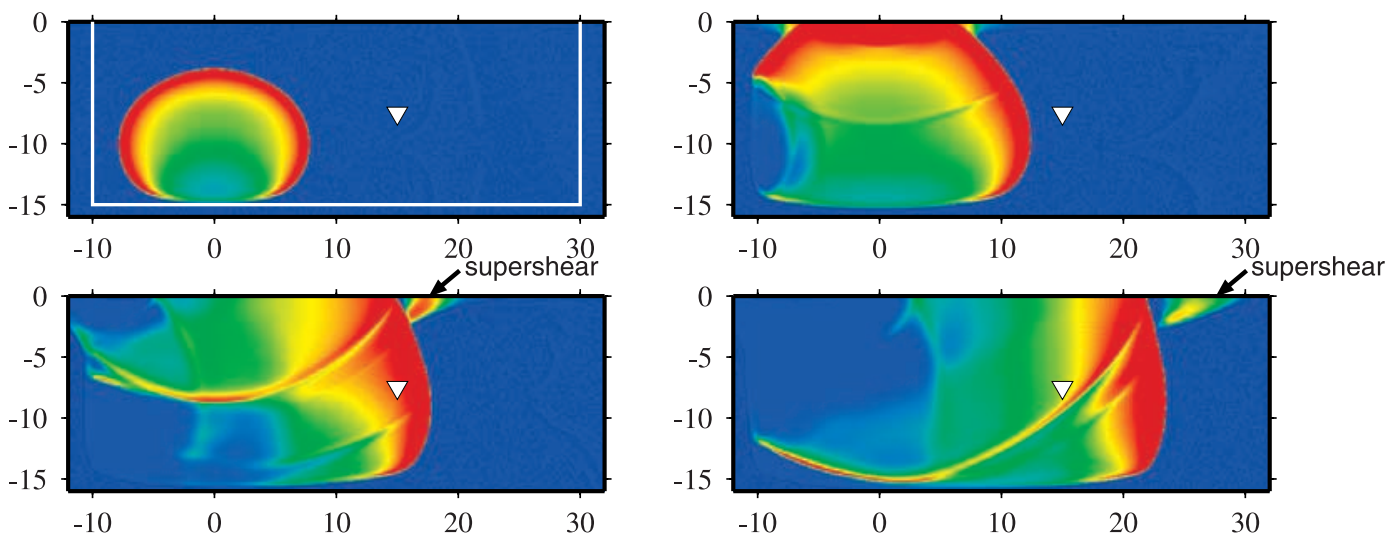

Case 2: With a shallow velocity-strengthening patch
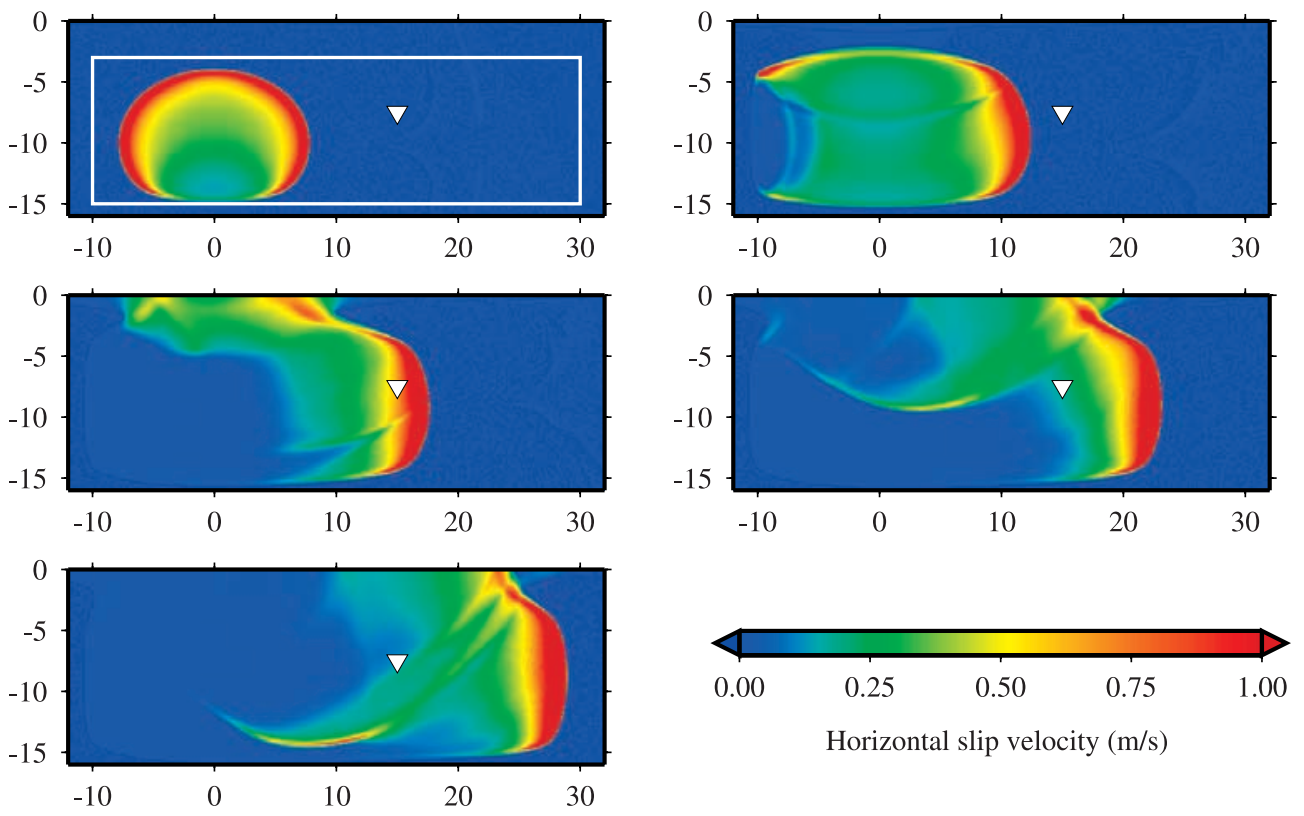

Figure 9. Snapshots of horizontal slip velocity $(\mathrm{m} / \mathrm{s})$ on the fault every 2 seconds for (top) case 1 and (bottom) case 2. White lines on two snapshots represent the boundary between velocity-weakening and velocity-strengthening regions. Slip velocity and slip at the location of an inverted triangle are plotted in Figure 12. Note that only a part of the fault close to the velocity-weakening region is shown.

[46] Figure 10 shows particle velocity seismograms on the free surface, $2.0 \mathrm{~km}$ away from the fault trace. We see that the arrival phase in the waveforms (a solid black line) has a speed higher than the shear wave speed $\left(V_{\mathrm{s}}\right)$. The differences in waveforms for the two cases are significant due to the combination of two effects in case 1: high slip velocity on the fault near the free surface and the supershear phase. The differences indicate that it might be possible to infer the existence of shallow velocity-strengthening patches from seismic observations.

\subsection{Smaller Final Slip Throughout the Fault}

[47] The shallow velocity-strengthening patch in case 2 causes an appreciable slip reduction over the entire fault (Figure 11). The existence of the shallow velocity-strengthening patch suppresses the slip due to the combination of two effects. The first one is that the healing phase created at the shallow rheological transition gradually ceases the slip at depth. The second effect is that the rupture reflected from the free surface in case 1 induces further slip at depths, whereas in case 2, the effect of the reflected rupture decreases because of the suppressed slip in the velocitystrengthening patch at shallow depths. Thus, the total amount of slip at depth is smaller in case 2 than in case 1 (i.e., $25 \%$ smaller at the receiver in Figure 11). The difference in final slip between case 1 and case 2 is largest next to the free surface. The resulting profile of slip with depth (Figure 11) indicates shallow coseismic slip deficit, which is qualitatively consistent with observations [Fialko et al., 2005]. Note that these two rupture scenarios yield comparable moment magnitudes; $M_{\mathrm{w}}=7.1$ in case 1 and 7.0 in case 2 . 
(A)

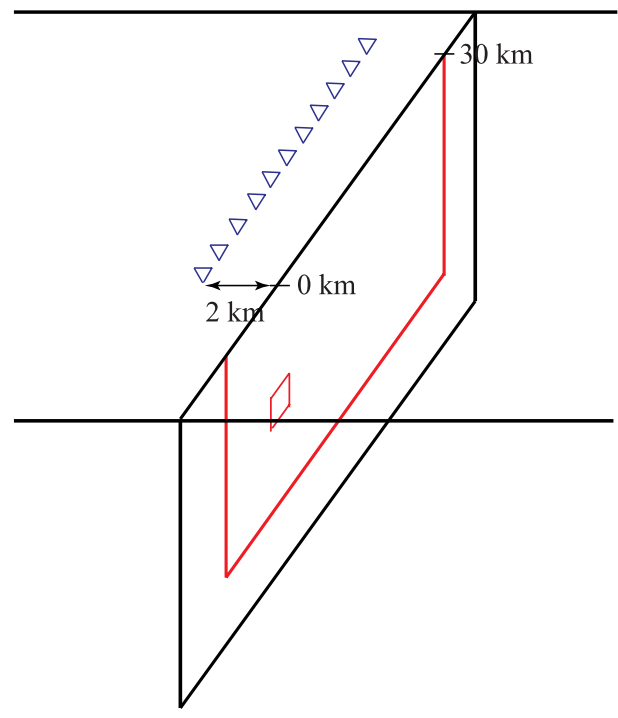

(B)

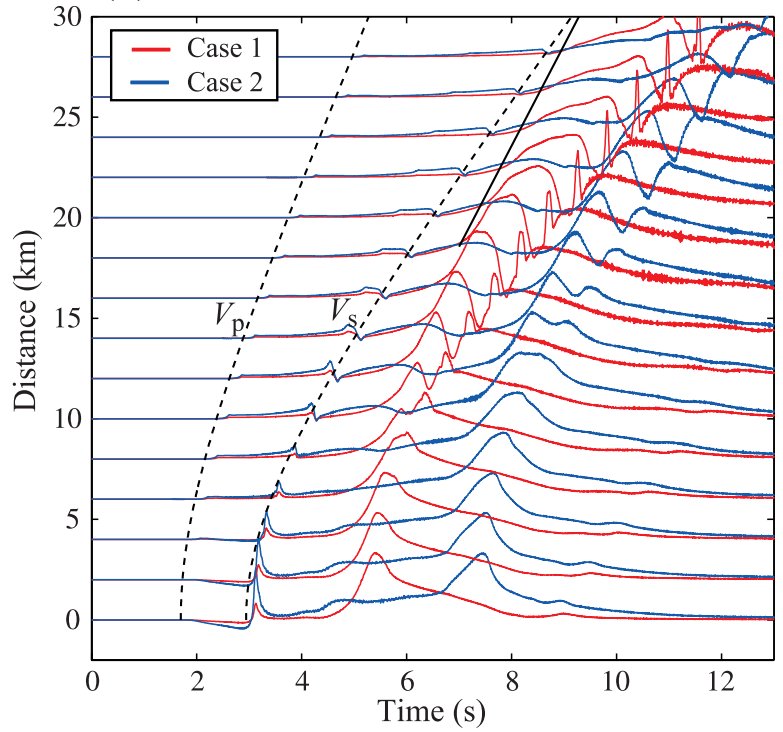

Figure 10. (a) Off-fault receiver locations for the 3-D SEM model. (b) Strike-parallel particle velocity at those receivers. The amplitudes at the individual receivers are normalized by the maximum amplitude at that receiver for case 1 (red curve). The dashed lines correspond to apparent $V_{\mathrm{p}}$ and $V_{\mathrm{s}}$ arrivals. The black solid line shows approximate arrival of the phase that corresponds to the supershear rupture.

[48] The slip reduction corresponds to smaller stress drop. The values of depth-averaged (from $0 \mathrm{~km}$ to $15 \mathrm{~km}$ ) static stress drop for cases 1 and 2 (Figure 11) are 4.9 MPa and 3.5 $\mathrm{MPa}$, respectively. Note that stress drop within the shallow velocity-strengthening patch is negative, that is, shear stress increases after the earthquake. Another mechanism that can contribute to negative stress drop at shallow depths is low initial stress in low-rigidity shallow materials resulting from uniform tectonic strain [Rybicki and Yamashita, 1998].
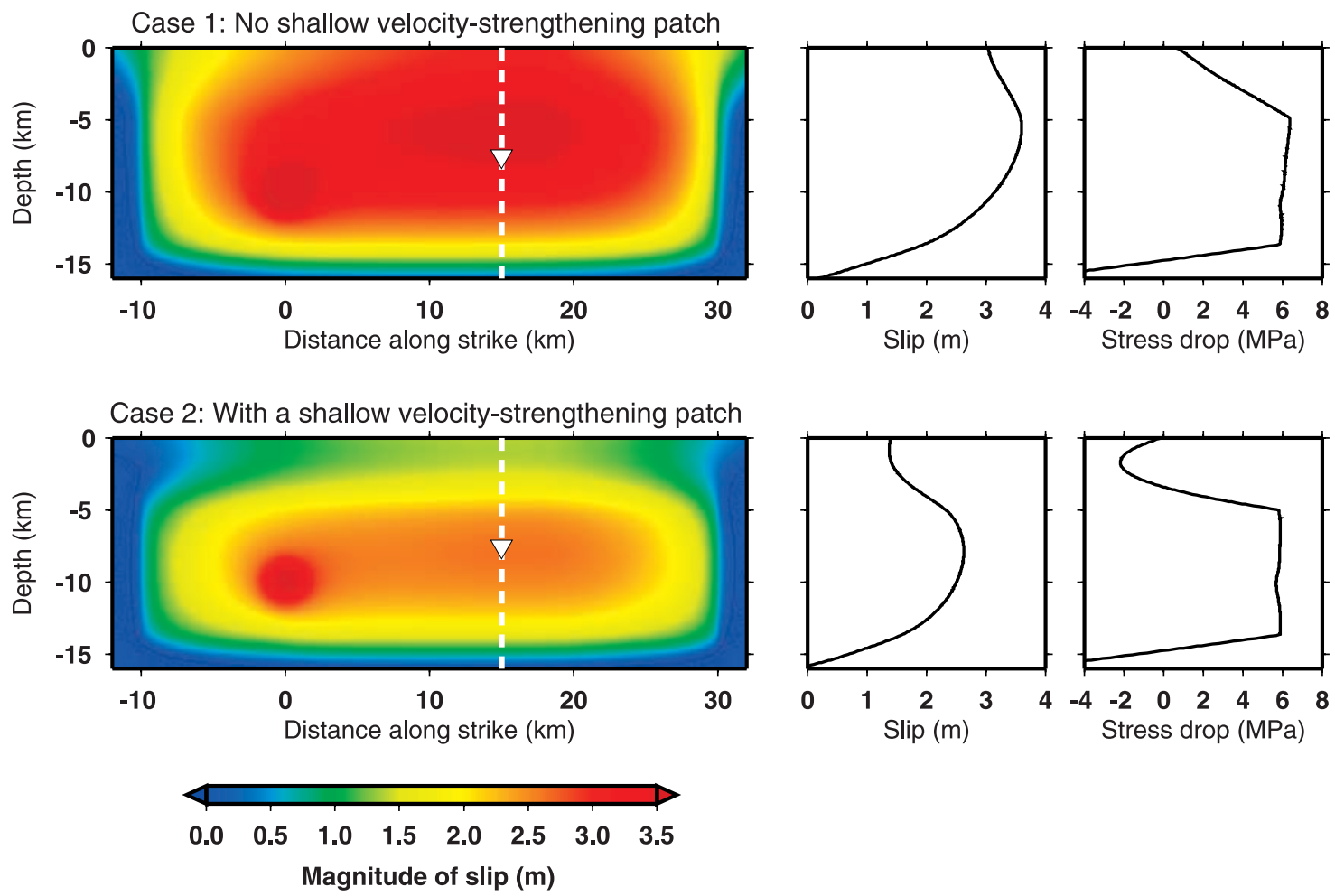

Figure 11. Final slip over the fault and depth profiles of slip and stress drop along the dashed lines for (top) case 1 and (bottom) case 2. Slip in case 2 is reduced throughout the fault. The shallow velocitystrengthening patch results in negative stress drop (or stress increase). 
(A)

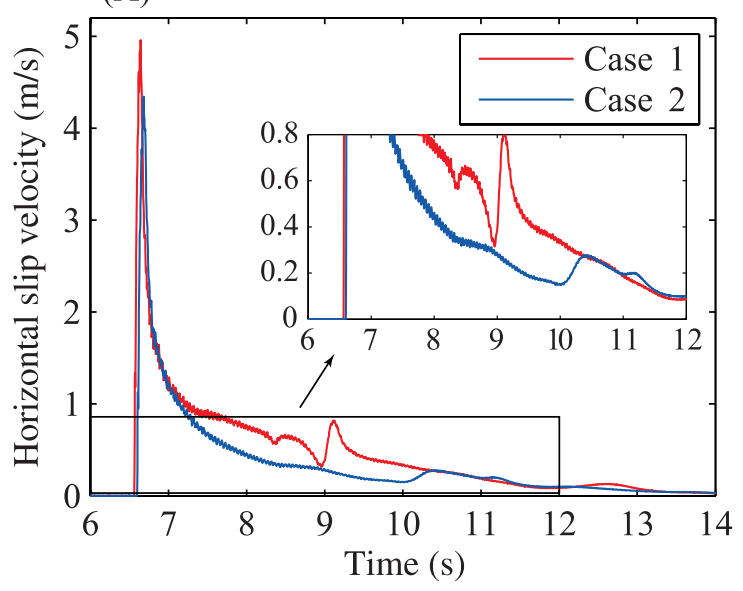

(B)

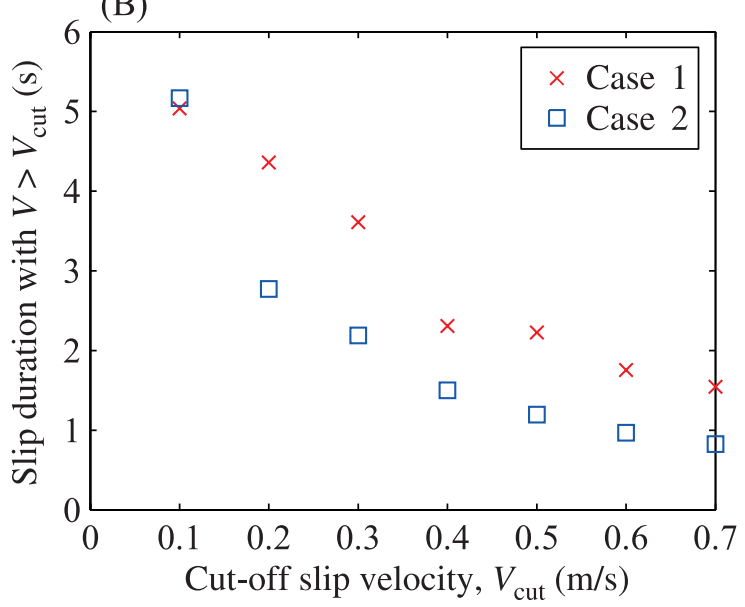

Figure 12. (a) Horizontal slip velocity at a location indicated by an inverted triangle in Figure 9 for cases 1 and 2. (b) Slip durations for cases 1 and 2 at the same receiver for different slip-velocity cutoffs. The slip durations are smaller for the case with the shallow velocity-strengthening region (case 2).

\subsection{Faster Decrease of Slip Velocity Behind the Rupture Front: Implications for the Risetime}

[49] One of the important source parameters inferred by seismic inversions is the risetime $T_{\mathrm{s}}$ (i.e., slip duration at a point on the fault). Let us consider the risetime for cases 1 and 2. Figure 12a shows the evolution of slip velocity at the middle of the seismogenic depth $(7.5 \mathrm{~km}$ depth) at the distance of $15 \mathrm{~km}$ from the center of the nucleation patch (the inverted triangle in Figure 9). This representative profile has a strong rupture front followed by gradually decreasing weak "tail", for both cases 1 and 2. Such slip velocity profile is commonly referred to as "crack-like," as there is no self-healing behind the rupture front. However, slip velocity in the "tail" is rather small and slowly varying compared to the rupture front. The risetime that seismic inversions would determine for such rupture should depend on the inversion method, the amount and quality of available seismic data, and other factors, and determining that "seismic" estimate of the risetime is beyond the scope of this study. To obtain some simple estimates, we consider here slip durations with slip velocities larger than a given cutoff value (Figure 12b). Note that the resulting slip durations are generally lower for case 2 which contains the shallow velocity-strengthening region.

[50] We compare these slip durations with the study by Heaton [1990] which demonstrated that the risetime $T_{\mathrm{s}}$ in seismic inversions is significantly shorter than the time $T_{\mathrm{s}}^{\mathrm{H}}$ required for rupture to receive the healing phase due to the effective seismogenic width of faults:

$$
T_{\mathrm{s}} \ll T_{\mathrm{s}}^{\mathrm{H}} \equiv \frac{2 \sqrt{S}}{3 V_{\mathrm{r}}},
$$

where $T_{\mathrm{s}}^{\mathrm{H}}$ is the risetime estimate from Day [1982] used by Heaton [1990] for the fault aspect ratio $<2, V_{\mathrm{r}}$ is the velocity of the rupture front, and $S$ is the ruptured area. Using the average rupture velocity around the receiver location in Figure $9(2.75 \mathrm{~km} / \mathrm{s})$ and the ruptured area up to the location $(25 \mathrm{~km}$ by $15 \mathrm{~km})$, we obtain $T_{\mathrm{s}}^{\mathrm{H}}=4.7 \mathrm{~s}$. This value is comparable to the slip duration in our simulations for the cutoff velocity of $0.1 \mathrm{~m} / \mathrm{s}$. For larger cutoff velocities, the estimated slip durations are shorter, especially for the case with the shallow velocity-weakening region, being closer to the range of risetimes given by seismic inversion data of Heaton [1990]. This is consistent with studies [e.g., Beroza and Mikumo 1996] that noted that fault heterogeneity can produce local arrest phases and reduce the risetime. The shallow velocity-strengthening region acts as such a heterogeneity, effectively reducing the width of the seismogenic zone, as well as diminishing the effect of the free surface. Note that a number of dynamic weakening mechanisms promote self-healing of ruptures and hence short risetimes [e.g., Lu et al., 2007, and references therein]; the work of Heaton [1990] advocated strongly ratedependent friction that can result from shear heating [e.g., Rice, 2006]. Our methodology can incorporate dynamic weakening mechanisms by combining them with RS friction as briefly discussed in section 6 .

\section{Effect of Velocity-Strengthening Friction at Shallow Depths on Ground Motion Amplification due to a Layered Bulk Structure}

[51] In this section, we investigate how low-velocity bulk layers at shallow depths, in combination with different fault rheologies, affect the ground motion. Figure 13a illustrates elastic parameters used for the layered bulk structure; two low-velocity layers above $5-\mathrm{km}$ depth are added to the homogeneous case used in section 4. This layered bulk model approximately corresponds to the 1-D Parkfield velocity structure used in the study by Custódio et al. [2005]. Uniform bulk attenuation is accommodated by the approach of Komatitsch and Tromp [1999], with the shear quality factor $Q_{\mu}=40$ and infinite bulk quality factor.

[52] We consider four earthquake scenarios. They are combinations of two different fault rheologies (velocity weakening up to the free surface versus a shallow $3-\mathrm{km}$ velocity-strengthening patch) and two different bulk structures (homogeneous versus layered). We make the seismic moment rate and total moment approximately equal in the 
(A)

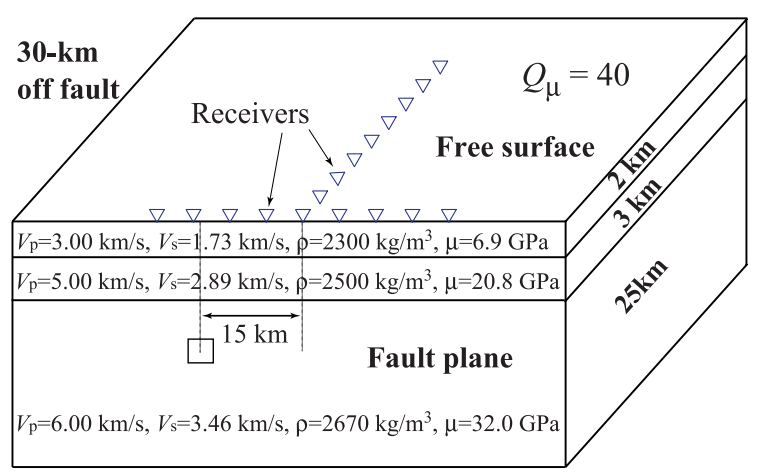

60-km along strike distance

(C)

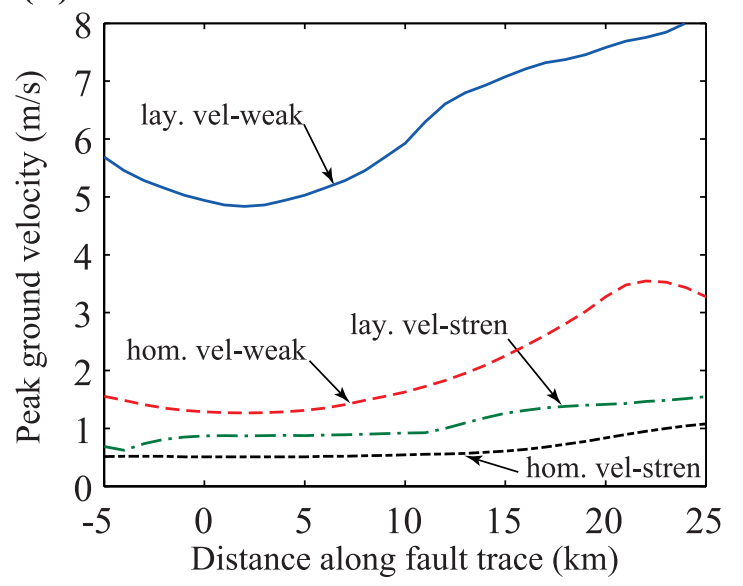

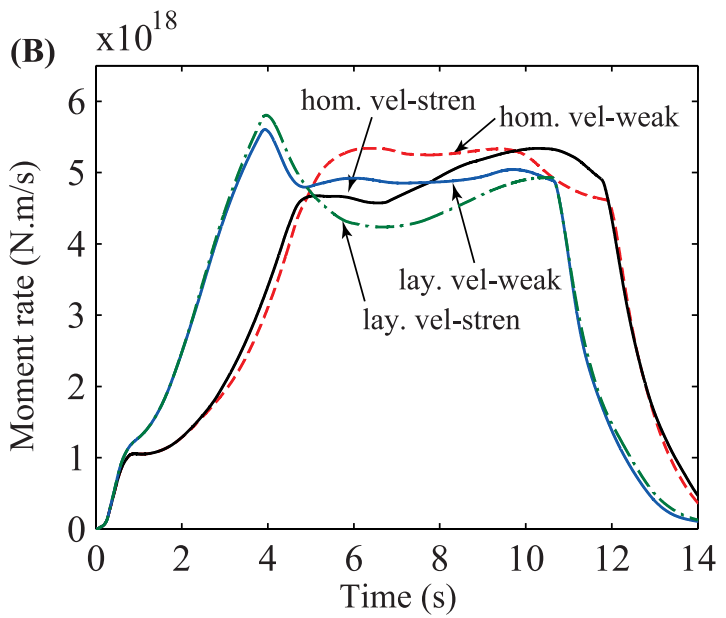

(D)

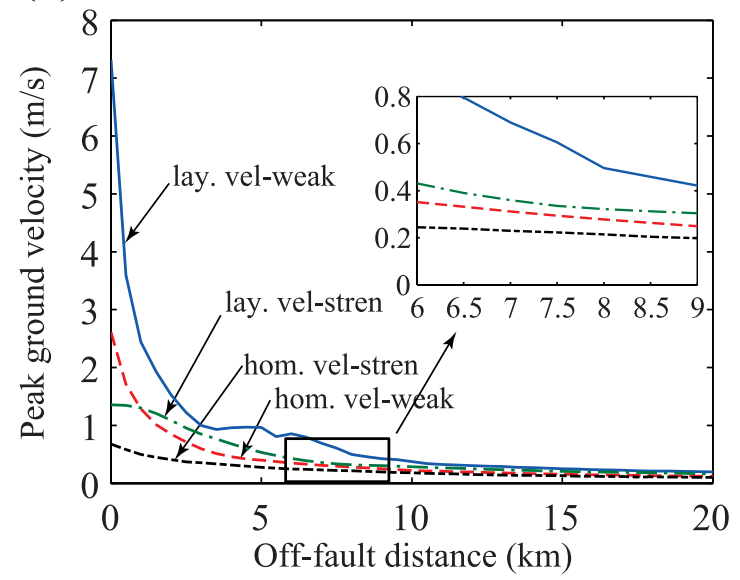

Figure 13. (a) A 3-D model with a layered bulk structure. (b) Computed moment rate for four different earthquake scenarios with (1) homogeneous bulk structure without the shallow velocity-strengthening patch, (2) homogeneous bulk structure with the shallow velocity-strengthening patch, (3) layered bulk structure without the shallow velocity-strengthening patch, and (4) layered bulk structure with the shallow velocity-strengthening patch. (c) Peak ground velocity (PGV), $\dot{u}_{\text {peak }}=\max \left(\sqrt{\dot{u}_{\mathrm{x}}^{2}+\dot{u}_{\mathrm{y}}^{2}+\dot{u}_{\mathrm{z}}^{2}}\right)$, at on-fault receivers $(z=0)$ shown in Figure 13a. PGV is much smaller in the cases with the shallow velocity-strengthening patch for both layered and nonlayered bulk. (d) PGV at off-fault receivers shown in Figure 13a. The difference in PGV for the different fault rheologies remains significant over off-fault distances comparable to the seismogenic width $(\sim 15 \mathrm{~km})$.

four cases to create similar source processes. As shown in section 4 , for a given initial stress, the case with the shallow velocity-strengthening patch results in smaller slip, and hence a smaller value of seismic moment, than the case with no shallow velocity-strengthening patch. To obtain similar values of seismic moment for the four scenarios considered here, we use, for the cases with the shallow velocity-strengthening patch, $3 \%$ larger initial shear stress than the one used in section 4. Figure 13b shows the computed moment rate for the four earthquake scenarios. We see that the four scenarios result in similar values of seismic moment rate; the total moment differs by less than $4 \%$. Note that the seismic moment is relatively insensitive to the change in a bulk structure because the slip gets amplified at places where the shear modulus is relatively low.

[53] The change in bulk properties as well as the slip redistribution due to fault rheology substantially influence the peak ground velocity (PGV). PGV at the on- and offfault receivers is amplified in the layered bulk cases due to the reduction in elastic moduli (Figures $13 \mathrm{c}$ and $13 \mathrm{~d}$ ). An interesting feature is the smaller values of PGV, for a given bulk structure, for the cases with the shallow velocitystrengthening patch, even as far as $10 \mathrm{~km}$ away from the fault. For example, the PGV at the $7.5-\mathrm{km}$ off-fault receiver is $45 \%$ smaller for the case with the shallow velocitystrengthening patch (blue line) than for the case with no shallow velocity-strengthening patch (green line). The difference is large near the fault trace and decreases with the distance away from the fault. This result suggests that both bulk structure as well as fault rheology are important parameters for the peak ground motion.

[54] Another interesting feature is the presence of highfrequency surface waves whose energy is trapped within the shallow low-velocity bulk layers (Figure 14). In the case of the layered bulk structure without the shallow velocitystrengthening fault rheology, high-frequency Rayleigh waves (Figure 14a) form as a result of the large faultperpendicular particle velocities on the fault near the free 

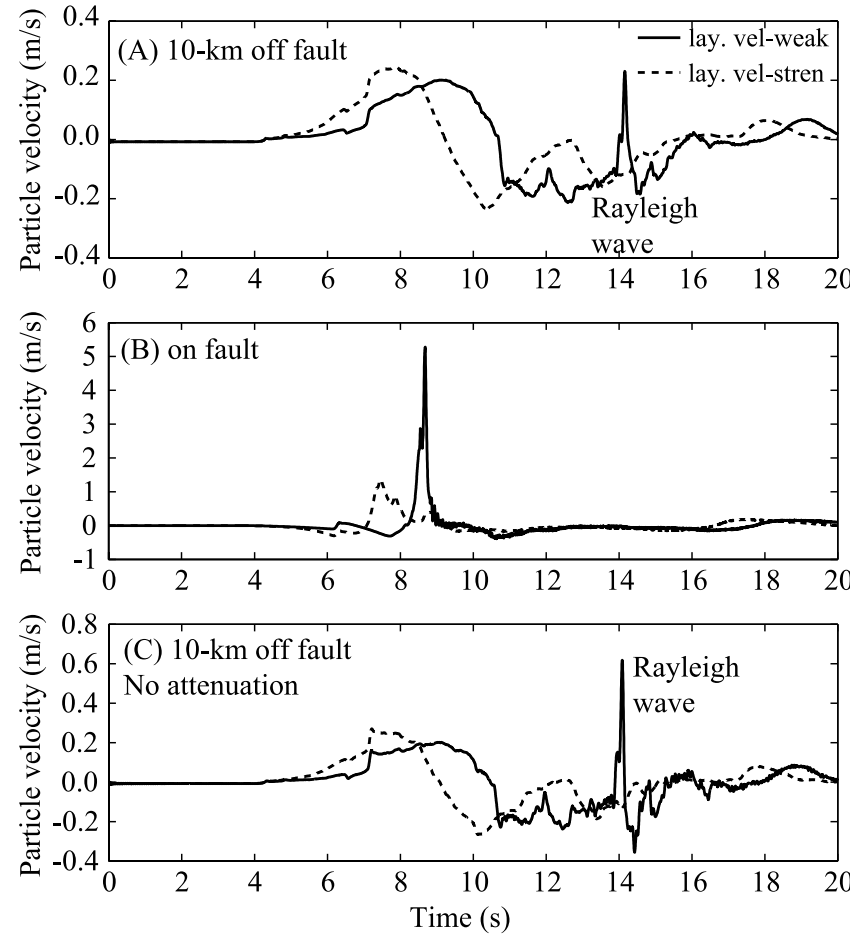

Figure 14. (a) Fault-perpendicular particle velocity at the off-fault receiver located $10 \mathrm{~km}$ away from the fault, at the distance of $15 \mathrm{~km}$ from the nucleation point along the strike. (b) Fault-perpendicular particle velocity at the on-fault receiver with the same along-strike distance. (c) Faultperpendicular particle velocity at the off-fault receiver in Figure $14 \mathrm{a}$ in the case without attenuation. These seismograms correspond to the cases with the layered bulk structure. When there is no velocity-strengthening region close to the free surface (solid lines in Figures 14a and 14c), a high-frequency Rayleigh peak is observed. In the nonattenuating medium (Figure 14c), the amplitude of the high-frequency Rayleigh wave becomes significantly higher than the body wave amplitude. Note that the scale is different in Figure 14a and 14c. The Rayleigh wave peak is not observed for the cases with the shallow velocitystrengthening patch both with and without attenuation (dashed lines in Figures 14a and 14c).

surface (Figure 14b), which are, in turn, related to the large slip velocities (e.g., fault-parallel velocities) near the free surface. In the absence of attenuation or for weaker attenuation $\left(Q_{s} \gtrsim 40\right)$, the amplitude of the Rayleigh waves becomes larger than the amplitude of body waves at the distance of several kilometers from the fault trace and remains high over a wide range of the off-fault distances. However, such high-frequency Rayleigh waves have not yet been observed in strong motion records (T. H. Heaton, personal communication, 2007). One explanation for the absence of the high-frequency Rayleigh waves is their scatter by more complex bulk structure than the idealized layered bulk assumed in our simulations (T. H. Heaton, personal communication, 2007). We show that a shallow velocity-strengthening fault region can provide an alternative explanation, since high-frequency Rayleigh waves do not form in the case with velocity-strengthening fault friction at shallow depths (Figure 14), which suppresses slip velocity near the free surface.

\section{Conclusions}

[55] We have developed an algorithm for simulating dynamic rupture on rate and state (RS) faults in the context of 2-D and 3-D SEM models. Our SEM test results have accuracy comparable to that of BIM over a wide range of node spacings. We have also demonstrated that the computational cost with respect to a given accuracy of a solution is smaller in calculations with the aging form of RS friction than with linear slip-weakening friction. Simulations with the slip form of RS friction require much higher numerical resolution than those with the aging form due to the higher maximum effective slip-weakening rate at the rupture front for the slip law than for the aging law, for the same rate and state parameters. As a result, we have found that solution errors in dynamic rupture problems are controlled by the resolution of the maximum effective slip-weakening rate at the propagating rupture front.

[56] Using the developed SEM approach, we have shown that the presence of velocity-strengthening fault friction at shallow depths significantly affects dynamic rupture. A shallow velocity-strengthening region suppresses supershear propagation near the free surface, which could explain the lack of universal observations of such near-surface supershear rupture. In addition, it decreases slip accumulation over the entire fault. The largest slip decrease occurs close to the free surface, consistently with the observed deficit of shallow coseismic slip in large earthquakes. Note that velocity-strengthening properties of the shallow layer can be adjusted to reduce the slip accumulation there further, or even completely prevent the rupture from propagating to the free surface. A shallow velocity-strengthening region results in faster decrease of slip at the tail of propagating rupture at seismogenic depths than in the case without such region, creating shorter effective risetimes. The amplification of ground motion due to low-velocity elastic structure is decreased in the presence of a velocitystrengthening region at shallow depths, because of the redistribution of slip. These results suggest the importance of the shallow velocity-strengthening fault region not only for quasi-static response such as aseismic transients and afterslip, but also for dynamic rupture and the associated seismic hazard.

[57] Note that earthquake rupture in this work is nucleated either abruptly or relatively rapidly, as common in simulations of a single earthquake [e.g., Harris et al., 2004; Day et al., 2005; Rojas et al., 2007]. While the conclusions in this study should not depend on the nucleation procedure, a number of earthquake problems require the ability to simulate more gradual nucleation under slow tectonic loading, postseismic and other aseismic slip, and sequences of simulated earthquakes, while still accounting for inertial effects during simulated earthquakes. For simple fault geometries and a uniform elastic medium, this has been accomplished by BIM approaches [e.g., Lapusta et al., 2000; Liu and Lapusta, 2006]. To allow for more flexibility in fault geometry and bulk properties in long-term simulations of fault slip, we are developing a combined SEM 
[Kaneko et al., 2007] that merges a quasi-static SEM with the fully dynamic SEM presented in this work.

[58] There is growing evidence that friction is much lower at seismic slip velocities than RS friction laws predict [e.g., Di Toro et al., 2003; Rice, 2006; Han et al., 2007; Lu et al., 2007, and references therein]. The presented SEM framework can be extended to include dynamic weakening mechanisms such as pore pressurization and flash heating [Rice, 2006, and references therein], which can be combined with RS friction to account for a wide range of seismic and aseismic slip velocities. SEM can incorporate complex 3-D geometrical effects such as 3-D basins and seismic Moho [Casarotti et al., 2007]. Furthermore, it can be extended to include nonplanar fault geometries and heterogeneous and/or nonelastic bulk properties, factors that are important for understanding fault behavior, and in particular large earthquakes.

\section{Appendix A: Fault Boundary Matrix}

[59] The fault surface $\Gamma$ consists of quadrilateral elements $\Gamma_{e}$ inherited from hexahedral elements lying on the two sides $\Gamma_{ \pm}$of the fault. The $\mathbf{B}$ in (1) is a sparse rectangular matrix obtained by assembling the contributions $\mathbf{B}_{e}$ from each of the fault boundary elements $\Gamma_{e}$ that are the same for the three components of traction. The term of $\mathbf{B}_{e}$ associated with the GLL node with local indices $(i, j)$ in $\Gamma_{e} \in \Gamma_{ \pm}$is

$$
B_{i j, i j}^{e \pm}= \pm \omega_{i} \omega_{j} j_{e}^{i j}
$$

where $\omega_{k}$ denote the weights associated with the GLL integration quadrature and

$$
J_{e}^{i j}=\left\|\frac{\partial \mathbf{x}}{\partial \xi} \times \frac{\partial \mathbf{x}}{\partial \eta}\right\|\left(\xi_{i}, \eta_{j}\right)
$$

is the Jacobian of the coordinate transformation from $\mathbf{x} \in \Gamma_{e}$ to $\boldsymbol{\xi}=(\xi, \eta) \in[-1,1]^{2}$.

[60] The outward normal vector of the fault boundary $\Gamma^{+}$ is obtained by

$$
\mathbf{n}(\xi, \eta)=\frac{1}{J_{e}} \frac{\partial \mathbf{x}}{\partial \xi} \times \frac{\partial \mathbf{x}}{\partial \eta} .
$$

\section{Appendix B: Rupture Initiation Procedure}

[61] To nucleate dynamic rupture in a short period of time, we need to abruptly increase slip velocity from values below typical plate loading rate $\left(\sim 10^{-12} \mathrm{~m} / \mathrm{s}\right)$ to coseismic ones $(\sim 1 \mathrm{~m} / \mathrm{s})$. To achieve this numerically, we use a perturbation of shear stress that smoothly grows from zero to its maximum amplitude $\Delta \tau_{o}$ over a finite time interval $T_{\text {ini }}$, and is confined to a finite circular region of the fault of radius $R$. Following the approach used by Rojas et al. [2007] and in the 2008 SCEC code validation, we apply a horizontal shear traction perturbation of the form

$$
\Delta \tau(x, z, t)=\Delta \tau_{o} F\left(\sqrt{\left(x-x_{o}\right)^{2}+\left(z-z_{o}\right)^{2}}\right) G(t)
$$

where

$$
F(r)= \begin{cases}\exp \left[r^{2} /\left(r^{2}-R^{2}\right)\right] & \text { if } r<R \\ 0 & \text { if } r \geq R\end{cases}
$$

and

$$
G(t)= \begin{cases}\exp \left[\left(t-T_{\mathrm{ini}}\right)^{2} /\left(t^{2}-2 t T_{\text {ini }}\right)\right] & \text { if } 0<t<T_{\text {ini }}, \\ 1 & \text { if } t \geq T_{\text {ini }} .\end{cases}
$$

The perturbation is radially symmetric, with the radial distance away from the hypocenter along the fault given by $r=\sqrt{\left(x-x_{o}\right)^{2}+\left(z-z_{o}\right)^{2}}$. We use $R=2.5 \mathrm{~km}, \Delta \tau_{o}=$ $19.0 \mathrm{MPa}$, and $T_{\mathrm{ini}}=0.1 \mathrm{~s}$.

[62] Acknowledgments. The authors thank Tom Heaton, Qinya Liu, and Yi Liu for helpful discussions. The reviews by Eric Dunham, Gaetano Festa, and an anonymous reviewer helped us improve the manuscript. We thank Eric Dunham for providing us with the unpublished functional form of the rupture initial procedure. Nadia Lapusta gratefully acknowledges the support of the National Science Foundation (grant EAR 0548277) and the Southern California Earthquake Center. SCEC is funded by NSF Cooperative Agreement EAR-0106924 and USGS Cooperative Agreement 02HQAG0008. Jean-Paul Ampuero was supported by SPICE, a Marie Curie Research and Training Network in the 6th Framework Program of the European Commission. The numerical simulations for this research were performed on Caltech Division of Geological and Planetary Sciences Dell cluster. This is SCEC contribution 1203 .

\section{References}

Aagaard, B. T., T. H. Heaton, and J. F. Hall (2001), Dynamic earthquake ruptures in the presence of lithostatic normal stresses: Implications for friction models and heat production, Bull. Seismol. Soc. Am., 91(6), $11,765-11,796$.

Ampuero, J.-P. (2002), Etude physique et numérique de la nucléation des séismes, Ph.D. thesis, Univ. Paris 7, Denis Diderot, Paris.

Ampuero, J.-P., and A. M. Rubin (2008), Earthquake nucleation on rate and state faults: Aging and slip laws, J. Geophys. Res., 113, B01302, doi:10.1029/2007JB005082.

Andrews, D. J. (1999), Test of two methods for faulting in finite-difference calculations, Bull. Seismol. Soc. Am., 89(4), 931-937.

Bayart, E., A. M. Rubin, and C. Marone (2006), Evolution of fault friction following large velocity jumps, Eos Trans. AGU, 87(52), Fall Meet. Suppl., Abstract S31A-0180.

Ben-Zion, Y., and J. R. Rice (1997), Dynamic simulations of slip on a smooth fault in an elastic solid, J. Geophys. Res., 102, 17,771-17,784.

Beroza, G. C., and T. Mikumo (1996), Short slip duration in dynamic rupture in the presence of heterogeneous fault properties, J. Geophys. Res., 101, 22,449-22,460.

Blanpied, M. L., D. A. Lockner, and J. D. Byerlee (1995), Frictional slip of granite at hydrothermal conditions, J. Geophys. Res., 100, 13,045-13,064.

Blanpied, M. L., C. J. Marone, and D. A. Lockner (1998), Quantitative measure of the variation in fault rheology due to fluid-rock interactions, J. Geophys. Res., 103, 9691-9712.

Casarotti, E., M. Stupazzini, S. J. Lee, D. Komatitsch, A. Piersanti, and J. Tromp (2007), CUBIT and seismic wave propagation based upon the spectral-element method: An advanced unstructured mesher for complex 3D geological media, in Proceedings, 16th International Meshing Roundtable, pp. 579-597, Springer, New York.

Chaljub, E., D. Komatitsch, J.-P. Vilotte, Y. Capdeville, B. Valette, and G. Festa (2007), Spectral element analysis in seismology, in Advances in Wave Propagation in Heterogeneous Media, Adv. Geophys., vol. 48, edited by R.-S. Wu and V. Maupin, pp. 365-419, Elsevier, New York.

Clayton, R., and B. Engquist (1977), Absorbing boundary conditions for acoustic and elastic wave equations, Bull. Seismol. Soc. Am., 67, 15291540

Custódio, S., P. Liu, and R. J. Archuleta (2005), The $2004 M_{w} 6.0$ Parkfield, California, earthquake: Inversion of near-source ground motion using multiple data sets, Geophys. Res. Lett., 32, L23312, doi:10.1029/ 2005 GL024417.

Day, S. M. (1982), Three-dimensional finite difference simulation of fault dynamics: rectangular faults with fixed rupture velocity, Bull. Seismol. Soc. Am., 72, 705-727. 
Day, S. M., L. A. Dalguer, N. Lapusta, and Y. Liu (2005), Comparison of finite difference and boundary integral solutions to three-dimensional spontaneous rupture, J. Geophys. Res., 110, B12307, doi:10.1029/ 2005JB003813

Day, S. M., S. H. Gonzalez, R. Anooshehpoor, and J. N. Brune (2008), Scale-model and numerical simulations of near-fault seismic directivity, Bull. Seismol. Soc. Am., 98, 1186-1206, doi:10.1785/0120070190.

Dieterich, J. H. (1978), Time-dependent friction and the mechanism of stick-slip, Pure Appl. Geophys., 116, 790-806.

Dieterich, J. H. (1979), Modeling of rock friction: 1. Experimental results and constitutive equations, J. Geophys. Res., 84, 2161-2168.

Dieterich, J. H. (1994), A constitutive law for rate of earthquake production and its application to earthquake clustering, J. Geophys. Res., 99, $2061-$ 2618.

Dieterich, J. H. (2007), Applications of rate- and state-dependent friction to models of fault slip and earthquake occurrence, in Treatise on Geophysics, vol. 4, pp. 107-129, Elsevier, Amsterdam.

Dieterich, J. H., and B. D. Kilgore (1994), Direct observation of frictional contacts: New insights for state-dependent properties, Pure Appl. Geophys., 143, 283-302.

Di Toro, Giulio, D. L. Goldsby, and T. E. Tullis (2003), Friction falls towards zero in quartz rock as slip velocity approaches seismic rates, Nature, 427, 436-438, doi:10.1038/nature02249.

Festa, G., and J. P. Vilotte (2005), The Newmark scheme as velocity-stress time-staggering: An efficient PML implementation for spectral element simulations of elastodynamics, Geophys. J. Int., 161, 789-812.

Festa, G., and J.-P. Vilotte (2006), Influence of the rupture initiation on the intersonic transition: Crack-like versus pulse-like modes, Geophys. Res. Lett., 33, L15320, doi:10.1029/2006GL026378.

Fialko, Y., D. Sandwell, M. Simons, and P. Rosen (2005), Three-dimensional deformation caused by the Bam, Iran, earthquake and the origin of shallow slip deficit, Nature, 435, 295-299, doi:10.1038/nature03425.

Gu, J.-C., J. R. Rice, A. L. Ruina, and S. T. Tse (1984), Slip motion and stability of a single degree of freedom elastic system with rate and state dependent friction, J. Mech. Phys. Solids, 32, 167-196.

Han, R., T. Shimamoto, T. Hirose, J.-H. Ree, and J. Ando (2007), Ultralow friction of carbonate faults caused by thermal decomposition, Science, 316, 878-881, doi:10.1126/science.1139763.

Haney, M., R. Snieder, J.-P. Ampuero, and R. Hofmann (2007), Spectral element modeling of fault-plane reflections arising from fluid pressure distributions, Geophys. J. Int., 170(2), 933-951.

Harris, R. A., et al. (2004), The source physics of large earthquakes: Validating spontaneous rupture methods, Eos Trans. AGU, 85(47), Fall Meet. Suppl., Abstract S12A-05.

Heaton, T. H. (1990), Evidence for and implications of self-healing pulses of slip in earthquake rupture, Phys. Earth Planet. Inter., 64, 1-20.

Hsu, Ya.-Ju., M. Simons, J.-P. Avouac, J. Galetzka, K. Sieh, M. Chlieh, D. Natawidjaja, L. Prawirodirdjo, and Y. Bock (2006), Frictional afterslip following the 2005 Nias-Simeulue earthquake, Sumatra, Science, 312, 1921 - 1926, doi:10.1126/science. 1126960

Ida, Y. (1973), The maximum acceleration of seismic ground motion, Bull. Seismol. Soc. Am., 63, 959-968.

Kaneko, Y., and N. Lapusta (2008), Variability of earthquake nucleation in continuum models of rate and state faults and implications for aftershock rates, J. Geophys. Res., doi:10.1029/2007JB005154, in press.

Kaneko, Y., N. Lapusta, and J.-P. Ampuero (2007), Spectral element modeling of dynamic rupture and long-term slip on rate and state faults, Eos Trans. $A G U, 88(52)$, Fall Meet. Suppl., Abstract S21B-0562.

Kato, N., and T. E. Tullis (2001), A composite rate- and state-dependent law for rock friction, Geophys. Res. Lett., 28(6), 1103-1106.

Komatitsch, D., and J. Tromp (1999), Introduction to the spectral-element method for 3-D seismic wave propagation, Geophys. J. Int., 139, 806822.

Komatitsch, D., and J. P. Vilotte (1998), The spectral-element method: an efficient tool to simulate the seismic response of 2D and 3D geological structures, Bull. Seismol. Soc. Am., 88, 368-392.

Komatitsch, D., S. Tsuboi, and J. Tromp (2005), The spectral-element in seismology, in The Seismic Earth, Geophys. Monogr. Ser., vol. 157, edited by A. Levander and G. Nolet, pp. 205-227, AGU, Washington, D.C.

Lapusta, N., and J. R. Rice (2003), Nucleation and early seismic propagation of small and large events in a crustal earthquake model, J. Geophys. Res., 108(B4), 2205, doi:10.1029/2001JB000793.
Lapusta, N., J. R. Rice, Y. Ben-Zion, and G. Zheng (2000), Elastodynamic analysis for slow tectonic loading with spontaneous rupture episodes on faults with rate- and state-dependent friction, J. Geophys. Res., 105, $23,765-23,789$

Liu, Y., and N. Lapusta (2006), Three-dimensional elastodynamic simulations of seismic and aseismic slip history of a planar strike-slip fault, Eos Trans. $A G U, 87(52)$, Fall Meet. Suppl., Abstract S34A-07.

Liu, Y., and J. R. Rice (2005), Aseismic slip transients emerge spontaneously in three-dimensional rate and state modeling of subduction earthquake sequences, J. Geophys. Res., 110, B08307, doi:10.1029/2004JB003424.

Lu, X., N. Lapusta, and A. J. Rosakis (2007), Pulse-like and crack-like ruptures in experiments mimicking crustal earthquakes, Proc. Natl. Acad. Sci. U.S.A., 104, 18,931-18,936.

Lyons, S. N., Y. Bock, and D. T. Sandwell (2002), Creep along the Imperial Fault, southern California, from GPS measurements, J. Geophys. Res., 107(B10), 2249, doi:10.1029/2001JB000763.

Madariaga, R., J.-P. Ampuero, and M. Adda-Bedia (2006), Seismic radiation from simple models of earthquakes, in Earthquakes: Radiated Energy and the Physics of Faulting, Geophys. Monogr. Ser., vol. 170, edited by R. Abercrombie et al., pp. 223-236, AGU, Washington, D.C.

Marone, C. (1998), Laboratory-derived friction laws and their application to seismic faulting, Annu. Rev. Earth Planet. Sci., 26, 643-696.

Marone, C. J., C. H. Scholz, and R. Bilham (1991), On the mechanics of earthquake afterslip, J. Geophys. Res., 96, 8441-8452.

Oglesby, D. D., R. J. Archuleta, and S. B. Nielsen (1998), Earthquakes on dipping faults: The effects of broken symmetry, Science, 280, $1055-$ 1059

Olsen, K. B. (2000), Site amplification in the Los Angeles basin from threedimensional modeling of ground motion, Bull. Seismol. Soc. Am., 90 S77-S94

Polet, J., and H. Kanamori (2000), Shallow subduction zone earthquakes and their tsunamigenic potential, Geophys. J. Int., 142, 684-702.

Rice, J. R. (1980), The mechanics of earthquake rupture, in Physics of the Earth's Interior, edited by A. M. Dziewonski and E. Boschi, Proc. Int Sch. Phys. Enrico Fermi, 78, 555-649.

Rice, J. R. (1993), Spatio-temporal complexity of slip on a fault, J. Geophys. Res., 98, 9885-9907.

Rice, J. R. (2006), Heating and weakening of faults during earthquake slip, J. Geophys. Res., 111, B05311, doi:10.1029/2005JB004006.

Rice, J. R., and Y. Ben-Zion (1996), Slip complexity in earthquake fault models, Proc. Natl. Acad. Sci. U.S.A., 93, 3811-3818.

Rice, J. R., and A. L. Ruina (1983), Stability of a steady frictional slipping, J. Appl. Mech., 50, 343-349.

Rojas, O., E. Dunham, S. Day, L. A. Dalguer, and J. Castillo (2007), Finite difference modeling of rupture propagation with strong velocityweakening friction, paper presented at SCEC 2007 Annual Meeting, South. Calif. Earthquake Cent., Palm Spring, Calif.

Rubin, A. M., and J.-P. Ampuero (2005), Earthquake nucleation on (aging) rate and state faults, J. Geophys. Res., 110, B11312, doi:10.1029/ 2005JB003686.

Ruina, A. L. (1983), Slip instability and state variable friction laws, J. Geophys. Res., 88, 10,359-10,370.

Rybicki, K. R., and T. Yamashita (1998), Faulting in vertically inhomogeneous media and its geophysical implications, Geophys. Res. Lett. 25(15), 2893-2896.

Seno, T. (2002), Tsunami earthquakes as transient phenomena, Geophys. Res. Lett., 29(10), 1419, doi:10.1029/2002GL014868.

Shearer, P., E. Hauksson, and G. Lin (2005), Southern California hypocenter relocation with waveform cross-correlation, part 2: Results using source-specific station terms and cluster analysis, Bull. Seismol. Soc. Am., 95(3), 904-915.

Vilotte, J., G. Festa, and J.-P. Ampuero (2006), Dynamic fault rupture propagation using nonsmooth spectral element method, Eos Trans. $A G U, 87(52)$, Fall Meet. Suppl., Abstract S52B-05.

J.-P. Ampuero and Y. Kaneko, Division of Geological and Planetary Sciences, California Institute of Technology, 1200 E. California Boulevard, MC 252-21, Pasadena, CA 91125, USA. (ampuero@gps.caltech.edu; ykaneko@gps.caltech.edu)

N. Lapusta, Division of Engineering and Applied Science, California Institute of Technology, 1200 E. California Boulevard, MC 104-44, Pasadena, CA 91125, USA. (lapusta@caltech.edu) 